Ann. Génét. Sél. anim., I980, 12 (I), 8I-IIo.

\title{
L'élevage en Corse : une bibliographie signalétique
}

\author{
J.J. LAUVERGNE, G. RAVIS-GIORDANI $\left({ }^{*}\right)$ et J.P. PARAIN $\left({ }^{* *}\right)$ \\ Département de Génétique animale, \\ Centre national de Recherches zootechniques, I.N.R.A., \\ 78350 Jouy-en-Josas \\ $\left(^{*}\right)$ Département de Sociologie et Ethnologie, \\ Université de Provence, \\ 29, avenue Robert-Schuman, \\ I3Ioo Aix-en-Provence \\ (**) Bibliothèque du Musée de l'Homme, Place du Trocadéro, \\ 75016 Paris
}

\section{Résumé}

Entre 1562 et 1979 on a recensé 3 I2 références se rapportant à l'élevage en Corse. Elles ont été produites par 434 auteurs différents. Les trois quarts des cotes datent des 30 dernières années et la moitié ont été écrites depuis 1970 . Cela dénote une accéleration marquée dans le dernier quart de siècle. La raison en est l'intérêt porté par les pouvoirs publics à l'aménagement de la Corse sur le plan agricole, économique et, maintenant, écologique. Les travaux récents, de mieux en mieux documentés, emanent d'une quarantaine d'organismes de recherches ou de développement.

\section{Introduction}

De nombreux chercheurs s'intéressent actuellement à la Corse dans toutes les disciplines agronomiques et le nombre des articles va se multipliant d'année en année. Des bibliographies signalétiques sont déjà parues aidant considérablement le chercheur mais elles gardent jusqu'à présent un caractère de généralité. Le temps d'une plus grande spécialisation semble maintenant venu. C'est ainsi que nous présentons une bibliographie signalétique consacrée à l'élevage en Corse. 


\section{I. - Matériel et méthodes}

\section{A. - Sources}

Nous sommes partis des bibliographies établies par StARACE (I943), BRUĠ̀RE (I974) et FIORI (I974, I975, I976) et du catalogue détaillé des articles parus dans le Bulletin de la Société des Sciences historiques et naturelles de la Corse (fasc. 598599, I977).

Nous avons complété ces compilations en consultant les services de documentation des personnes et institutions suivantes :

- Archives départementales de la Corse du Sud (Ajaccio).

- Association pour 1'Étude Écologique du Maquis (A.P.E.E.M.) (Bastia).

- Centre Beaubourg-Georges Pompidou (Paris).

- Centre d'Études Corses (C.F.C.), Université de Provence (Aix-en-Provence).

- Centre d'Études Phytosociologiques et Écologiques L. EMBERGER (C.E.P.E.) (B.P. 505I Montpellier).

- Centre National de Recherches Zootechniques (C.N.R.Z.) (Jouy-en-Josas).

- Centre de Promotion Sociale (C.P.S.) (Corte).

- Centre Régional de Documentation Pédagogique (C.R.D.P.) (Ajaccio).

- Département de Génétique animale, C.N.R.Z. (Jouy-en-Josas).

- Ecole Nationale Vétérinaire d'Alfort.

- François FloRI, Bastia (Documentation personnelle).

- Maison Nationale des Éleveurs, (M.N.E.) (Paris).

- Ministère de 1'Agriculture, Rue de Varenne (Paris).

- Musée de 1'Homme (Paris).

- Société pour la mise en valeur de la Corse (S.O.M.I.V.A.C.) (Bastia).

- Ville d'Ájaccio (Bibliothèque Municipale).

Nous avons en outre fait appel au fichier électronique de LOOCKED Californie : Animal Breeding et de 1'I.A.E.A. de Vienne (Autriche) : Agris.

Les bibliographies, parfois fort complètes d'ouvrages académiques comme ceux de SIMI (I966) ou ÉTIENNE (I977) ont été également consultées avec fruit.

\section{B. - Vérifications}

Dans leur grande majorité, les références ont été vues par au moins l'un des auteurs. Il en est pourtant un certain nombre pour lesquelles on a dû se fier au titre ou se rapporter à la vigilance des auteurs qui les ont citées. Il s'agit, soit de références anciennes et difficilement retrouvables, soit de cotes des Archives pour lesquelles on n'a pas eu le temps matériel de procéder à toutes les vérifications.

\section{C. - Mode de présentation}

\section{Référenciation}

Pour certains articles, il est parfois difficile de trouver la séquence auteur, date, titre, éditeur ou journal, soit que l'une ou l'autre de ces mentions manquent ou qu'il soit difficile de distinguer entre l'auteur et l'éditeur. On s'est efforcé de faire pour le mieux en évitant, autant que possible, de quoter des auteurs anonymes. 
Pour cette référenciation, on est également confronté au problème des abréviations qu'on s'est efforcé de résoudre en donnant une liste que le lecteur pourra vérifier et compléter pour certains détails en consultant le dictionnaire de FAURE (I978).

\section{Présentation proprement dite}

Comme pour une précédente bibliographie (LAURANS et LAUVERGNE, I979), on donne les références chronologiquement en assortissant cette liste d'un index " auteurs " et d'un index " matières".

\section{Commentaires}

Après la liste bibliographique et les index, on s'est efforcé dans une brève discussion de dégager la dynamique des recherches sur l'élevage en Corse, en se basant sur quelques statistiques simples et de souligner les lacunes de la présente liste.

\section{Liste des abréviations ou sigles}

A.D.I.R.
ALIVI

A.P.E.E.M.

A.R.E.E.A.R.

A.T.R.S.P.

A.T.P.

B.D.P.A.

C.D.J.A.

C.E.P.E.

C.E.R.A.F.E.R.

C.E.R.E.S.M.

C.E.T.A.

C.N.R.A.

C.N.R.S.

C.N.R.Z.

C.P.S.

C.R.D.P.

C.T.C.P.

D.D.A.

D.E.S.

D.G.R.S.T.

D.S.A.

D.S.V.

E.D.E.

E.N.S.A.

E.S.I.T.P.A.

F.A.O.

F.D.S.E.A.

G.R.

G.R.E.S.E.

G.V.A.

I.N.A.E.A.

I.N.R.A.

M.A.B.
Atelier de Documentation et d'Information rurales.

Nom de l'Association pour le Développement Économique et Culturel de la Région de Sollacaro (ALIVI = oliviers).

Association pour l'Étude Écologique du Maquis.

Atelier Régional d'Études Économiques et d'Aménagement rural.

Arts et Traditions populaires.

Bureau pour le développement de la Production Agricole.

Centre des Jeunes Agriculteurs.

Centre d'Études Phyto-sociologiques et Écologiques (C.N.R.S. Montpellier)

Centre d'Etudes et de Recherches Ethnologiques sur les Sociétés Méditerranéennes (Aix-en-Provence).

Centre d'Étude Technique Agricole.

Centre National de Recherhces Agronomiques (I.N.R.A., Versailles). Centre National de la Recherche Scientifique.

Centre National de Recherches Zootechniques (I.N.R.A., Jouy-en-Josas). Centre de Promotion Sociale (Corte).

Centre régional de Documentation Pédagogique.

Direction Départementale de l'Agriculture.

Diplôme d'Études Supérieures (Université).

Délégation Générale à la Recherche Scientifique et Technique.

Direction des Services Agricoles (ancien nom de D.D.A.).

Direction des Services Vétérinaives.

Établissement Départemental d'Élevage.

École Nationale Supérieure Agronomique (Toulouse).

École Supérieure Institut Technique de Pratique Agricole (Paris).

Food and Agriculture Organisation (Rome), en français O.N.U.A.A.: dépend de l'O.N.U.

Fédération Départementale des Syndicats d'Exploitants Agricoles. Génie rural.

Groupe de Recherches sur l'Espace, la Société et l'Environnemcnt.

Groupement de Vulgarisation Agricole.

International Atomic Energy Agency (Vienne, Autriche).

Institut National de la Recherche Agronomique.

Man and Biosphere (Organisation Écologique Internationale). 
M.I.P.A.E.N.M.

O.N.F

O.N.U.A.A. ou O.A.A. Organisation pour l'Agriculture et l'Alimentation (Rome), dépend des P.U.F.

S.A.G.A.

S.C.E.E.S.

S.C.E.T.

S.E.E.E.E.

S.E.I.

S.E.T.C.O.

S.O.D.E.T.E.G.

S.O.M.I.V.A.C.

S.R.A.F.

S.R.S.A.

S.S.D.

S.U.A.D.

Mission Interministérielle de la Protection et de l'Aménagement de l'Espace Naturel Méditerranéen.

Office National des Forêts.

Nations Unies (en Anglais F.A.O.).

Presses Universitaires de France (Éditeur, Paris).

Station d'Amélioration Génétique des Animaux (I.N.R.A., 'Toulouse). Service Central des Enquêtes et Êtudes Statistiques (Ministère de 1'Agriculture).

Société Centrale pour l'Équipement Touristique (9o bd de la Tour Maubourg Paris).

\author{
Service d'Expérimentation et d'Information (I.N.R.A.). \\ Société pour l'Équipement touristique de la Corse. \\ Société d'Études Techniques et d'Entreprises Générales. \\ Société pour la Mise en Valeur agricole de la Corse (Bastia). \\ Service Régional d'Aménagement forestier. \\ Service régional de Statistique agricole.
}

Service d'Utilité agricole et de Développement (Chambres d'Agriculture).

\section{Liste chronologique}

$$
\text { I } 562
$$

I. ARChives dífPartementales DE LA CORSE DU SUD, 1562. Série C Ropale p. 42 (relatif à la clôture).

$$
1635
$$

2. ARchives dU Sénat DE GÈNEs (Filza cultivationum), 1635. Décrets (les terres non clôturées du Nebbio sont considérées comme des terres de parcours). Copie Arch. Départ. Corse $d u S u d, 99$ et II6.

$$
\text { I638 }
$$

3. Libro delife piante, 1638 . Ordre du 31 janvier 1638 du Commissaire F. Negroni. Manuscrit, Bibliothèque d'Ajaccio.

\section{I69I}

4. ARchives DÉpartementales de La CORSE du Sud, i691. Série C. p. 527 (droit de pacage dans le Nebbio).

$$
1770
$$

5. Archives départementales de la CORSE du Sud, i77o. Ceppi du Notaire FalConetrir de Castrfao, Arch. Dep. de la corse du Sud Ser. O. Castifao.

6. ARChives NATIONALES, I 77o. Mémoires et documents sur l'établissement des prairies naturelles ou artificielles et un plan pour rendre la Corse commerçante. Arch. Nat. Paris, Série QI, 29I.

$$
\text { I77I }
$$

7. ARChIVes Départementales DE LA CORSE DU Sud, I77I. A propos de la règlementation du libre parcours. Arch. Dép. de la Corse du Sud. B i4 et B 63.

8. CODE CORSE, I 77I. Concernant le mesus champêtre. t. II, 372. 
1774

9. Archives nationales, I 774. Mémoires et tabelles concernant l'établissement des pâtres et bergers de la partie orientale de l'isle de Corse compris seulement entre Tavignano et Fiumi Orbo et l'entre Fiumi Orbo et le Trare et les monts de Sarri de Gonca et de Lecci, jusque vers la rivière de Ste-Lucie, par ordre de M. le Comte de Marbeuf. Arch. nat. Paris, série $\mathrm{K}$, I 229 , I, VII, I 5 .

$$
1785
$$

IO. Fizelikere (DE LA), I 785. Mémoire sur la province ou juridiction de Sartène ou de la Rocca. Arch. Nat., K I $228 \mathrm{n}^{\circ} 39$, et in Corse historique, I 963.

\section{I795}

i i. Plan terrier de ra Corse. Arch. Dép. de la Corse du Sud.

$$
\text { I798 }
$$

I2. FEYDEL, I 798. Mœurs et coutumes des Corses. Garnery, Paris (an VII), 182 p.

\section{802}

13. Pietrry A., I802. Statistique du département du Golo par le citoyen Pietrry, Préfet. Publié par ordre du Ministère de l'intérieur. Imp. Le Clère, Paris (An IX).

$$
\text { I803 }
$$

I4. ARChIVES DÉPARTEMENTALES DE LA CORSE DU SUD, I803. Questionnaire adressé aux maires par le préfet du Golo en r803 sur l'assolement. Arch. Dép. série I3 M carton 2, cité par P. SrMI.

I5. TESSIER C., I803. État où était l'agriculture dans l'isle de Corse en r786. Ann. Agric. Paris, I4.

\section{8 I 2}

I6. ARchives DÉPARTementales DF LA Corse DU Sud, I8I2. Statistiques équestres. Arch. Dép. de la Corse du Sud, GM 874.

I 8 I 3

17. ARChIVES DÉPARTEMENTALES DE LA CORSE DU SUd, I8I2-I8I3. Statistiques bovines, Archiv. Dép. de la Corse du Sud, GM 876.

\section{8 I 8}

18. ARChIVES DÉPARTEMENTALES DE LA CORSE DU SUD, I8I8. Circulaires à MM. les maires relatives au parcours, du 20 Août I8I8. Avch. Dép. de la Corse du Sud.

\section{I $82 \mathrm{I}$}

19. ARchives DÉPARtementales DE LA CORSE DU SUd, I 82 I. Questionnaire chèvre. Arch dép. de la Corse du Sud, GM 879.

$$
\text { I } 824
$$

20. Vigarous, I824. Note succincte sur l'état actuel de l'agriculture en Corse. Bull. Soc. Agr. Hérault, Montpellier, 9 


$$
\text { I } 825
$$

21. Archives dípartementales de la CORSe du Sud, i825. Circulaire du préfet Lantivy. sur les parcours. Arch. dép. de la Corse du Sud.

\section{828}

22. ARchives DFPARTEMENTALES DE LA CORSE DU SUd, 1828. Statistiques laines et bêtes, I789-1828. Arch. Dép. de la Corse du Sud, GM 878 .

\section{829}

23. ARChives DÉpartementales De LA CORSE DU SUd, 1829. Enquête agricole de i 829 . Arch. Dép. de la Corse du Sud, $\mathrm{n}^{\circ} 36$.

24. Paléologue G., I829. Agriculture de la Corse, Lettres écrites à M. Fantier par M.G.P., Ann. agric. de Roville, Paris.

$$
\text { I } 835
$$

25. Carlotti R., i835. De l'état de l'agriculture en Corse et des moyens de l'améliorer. Mém. Soc. imp. Agric., Paris, 422.

26. RoBIQUET F. I 835, Recherches historiques et statistiques sur la Corse. Paris et Rennes, 399-40 I, 593.

$$
\text { I } 837
$$

27. Mol, L., I837. Agriculture de la Corse. Agric. prat. Jardin. Écon. domest. Paris I.

$$
\text { I } 840
$$

28. BLANQui F. A. J., I840. Rapport sur l'état économique et moral de la Corse en i839. Didot frères, Paris, $84 \mathrm{p}$.

29. Gavini, I840. De l'agriculture de la partie orientale de la Corse.

3o. Ḿ́riméte P., I 840 . Notes d'un voyage en Corse. Paris.

$$
\text { I } 842
$$

3r. Gavini A. J., I842. Analyse statistique et considérations d'économie rurale sur la Corse. Thiers (Bibliothèque de Bastia).

$$
\text { I } 843
$$

32. Statuti CIVILI E CRIMINALI Di CORSICA, I843. Publicati con addizione inedite e con una introduzione. Lyon (Bibliothèque de Bastia et Avch. Dépt. de la Corse du Sud).

$$
\text { I845 }
$$

33. CARLotri R., i 845. De l'état de la police rurale et de l'agriculture en Corse. Journal de la Corse, $\mathrm{I} 8-2-1845$.

34. Pozzo di borgo C. A., i 845 . Un mot sur les moyens d'améliorer l'agriculture en Corse. Imp. G. Marchi, Ajaccio.

$$
\text { I846 }
$$

35. Weipert A., i846. Mémoire sur la culture du mûrier en Corse. Paris.

$$
\text { I849 }
$$

36. Weipert A., I849. Conseil aux producteurs de soie en Corse. Goetshy, Altkirch. 
1852

37. Bonne A., 1852. Fabrication du bruccio (sic) ou fromage corse. J. Agric. prati. Jardin. $3^{\mathrm{e}}$ série, IV, Maison rustique du XIXe siècle, Paris $2^{*}$ partie.

\section{853}

38. CARLOTTI R., I853. Les chèvres, comment parvenir à leur cantonnement. Journal de la Corse, $\mathrm{I} 8$-I.

39. Cart,otri R., I853. Des biens communaux en Corse. Journal de la Corse, 4/r, I5/2, $22 / 2$.

40. Marchi G., 1853. Quelques réflexions sur l'état actuel de l'avenir de l'Agriculture en Corse. Imp. Marchi. Ajaccio.

$$
\text { I } 854
$$

4I. Carlotiti R., I854. Quels seront les résultats de la loi du 22 juin i 854 qui supprime la servitude du parcours et abolit le droit de veine pâture en Corse. Journal de la Corse.

42. CARLOT'Tr R., I854. Traité élémentaire de pratique agricole à l'usage spécial des cultivateurs de la Corse. Imp. Marchi, Ajaccio, 94 p.

\section{855}

43. BuRnouf, 1855. Rapport sur l'amélioration de la race bovine - Amélioration de la race cavalière - Haras, fait à la Société d'Agriculture de Corse en I854. Ann. Agric., Corse, Bastia. 59, 96 .

44. PoLI, I855. Rapport sur le moyen le plus propre à améliorer la race ovine Corse sous le double rapport de la taille et de la toison fait àla Société d'Agriculture d'Ajaccio le 13/8/1 854 . Ann. Agric., Corse, Bastia 89-96.

$$
\text { r } 856
$$

45. Foata (Abbé de la), I856. Du parcours et de la vaine pâture. Fabiani, Bastia.

\section{857}

46. Griscelili DE Vezzani J. F., i 857. Lettre d'un berger Corse à S.M. L'Empereur des Français (sur le parcours et la vaine pâture). Boucquin, Paris.

\section{$\mathbf{1} 858$}

47. Maestroni-Meglia, i 858 . Recueil des usages locaux d'Ajaccio. Ajaccio.

\section{2}

48. Archives départementales de la Corse dU Sud, i862. Consommation de viande 1836-1862. Arch. Dép. de la Corse du Sud, GM 884.

$$
\text { I } 863
$$

49. Terigi P., 1863. Mémoire sur la fabrication des fromages en Corse. Imp. Garcin, Draguignan.

$$
\text { I } 864
$$

50. Limperani J. A. de, i864. Rapport sur le concours agricole qui a eu lieu à Bastia le 30 décembre 1863 . Imp. Fariani, Bastia, $30 \mathrm{p}$. 


\section{865}

5I. Prasseto, i865. Rapport sur l'état de l'agriculture en Corse lu à la séance de la Soc. d'agriculture d'Ajaccio le 4 juin 1865. Imp. Marchi, Ajaccio.

\section{867}

52. Abbatucci S., I 867 . Enquête agricole. Imp. impériale, Paris.

53. ENQUÊTE AGRICOLE, I 867 . Enquêtes départementales, $28 \mathrm{e}$ circonscription : Corse. Imp. impériale, Paris.

54. Limperani J. A. de, i 867 . Rapport sur les résultats de l'enquête agricole et la situation de l'agriculture à la fin de l'année I 866 . Imp. Fabiani, Bastia, $3^{8} \mathrm{p}$.

\section{869}

55. Cantal (R. du), i 869. Rapport sur la production animale du département de la Corse et spécialement sur sa race de chevaux. Imp. Lecca, Ajaccio.

$$
\text { I } 870
$$

56. Otravi G. A. I87o. Il presente e l'avenire dell' agricultura in Corsica. Il coltivatore.

$$
\text { I } 874
$$

57. Pozzo di Borgo C. A., I874. Aperçu agricole de la Corse et guide de la bonne culture. Imp. Lecca, Ajaccio.

$$
\text { I } 876
$$

58. Carlotri R., r 876 . Du parcours et de la vaine pâture en Corse. Imp. Lecca, Ajaccio.

$$
\text { I } 877
$$

59. BeLLon, I877. Essai sur la production animale de la Corse. Mémoire conservé par la Société nationale et centrale de Médecine Vétérinaire.

6o. CarlotTI R., I877. Les moyens de développer l'industrie séricicole en Corse. Imp. Leca, Ajaccio.

$$
\text { I } 889
$$

6r. BIGOT M., ESCARD F., I889. Paysans corses en communauté : porchers - bergers des montagnes de Bastelica. Propriétaires ouvriers dans le système de travail sans engagement d'après les renseignements recueillis sur les lieux en I 869; les Ouvriers des deux Mondes, Firmin Didot, Paris, réed. Il Muntese. Bastia, I971, 73 p.

$$
\text { I } 893
$$

62. ARCHIVES DÉPARTEMENTALES DE LA CORSE DU SUd, i893. Enquête de consommation par commune 1893. Arch. Dép. de la Corse du Sud, GM 953.

63. BERGERAT E., I893. La chasse au Mouflon ou petit voyage philosophique en Corse. Paris.

64. VullLIER G., I 893. Les îles oubliées : Baléares, Corse, Sardaigne. Hachette, Paris.

$$
\text { I } 898
$$

65. Ardjuin - Dumazet, i898. Voyage en France, La Corse. Berger Le vrault, Nancy. 


\section{905}

66. Larue P., I905. Aperçu agronomique sur la Corse. Le Progrès agricole et viticole, Montpellier.

$$
\text { I } 909
$$

67. Carrier J., igo9. Enquête sur la vie pastorale. La Corse agricole, sept-oct.

68. RAVEL, L., I909. L'agriculture en Corse. Sarre et Roumegous, Montpellier.

$$
\text { IgIo }
$$

69. Sanson A., igro. Traité de Zootechnie. IV. Zoologie et Zootechnie spéciales, Bovidés, Taurins, Bubalins et Zébus. Librairie agricole de la Maison Rustique, Paris, 139.

$$
\text { I9II }
$$

7o. Ferton C., 191 I. Sur quelques produits de l'industrie laitière en Corse. C.R. Assoc. Fr. Avanc. Sci., $4^{\mathrm{o}}$ session, Dijon. XC, ${ }^{\mathrm{re}}$ partie, 1030-1040.

$$
\text { I9I2 }
$$

7r. Giraud - Genet L., igi2. Le régime pastoral de la Corse. Imp. Hacques, Besançon.

$$
\text { I9I4 }
$$

72. Blianchard R., I9I4. Les genres de vie en Corse et leur évolution. Rec. trav. Inst. géogr. alp. II, 187-238 et Bull. Soc. Sci. hist: nat., Corse, 1915 (364-366), 5-96.

\section{I9I5}

73. Chorn P. de, igr6. La situation chevaline en Corse. Paris, 8 p.

$$
\text { I9I8 }
$$

74. ANFossi G., I918. Recherches sur la distribution de la population en Corse. Rec. trav. Inst. Géogr. Alp.

$$
\text { I } 920
$$

75. Piobb, (Comte P. Vincentr), r92o. La Corse d'aujourd'hui, ses mœurs, ses ressources, sa détresse. Paris.

$$
\text { I } 921
$$

76. Natali J. B.; i921. Nos géorgiques. Bull. Soc. Sci. hist. nat., Corse., 41 (429-432), I-86.

77. Pompei P. I921. État actuel de la Corse. Paris.

$$
\text { r } 922
$$

78. BOYER L., 1922. L'industrie du Roquefort' en Corse, La Corse agricole, I ${ }^{\text {er }}$ janvier.

79. BOYER L., SAjous P., 1922. L'amélioration de la race ovine en Corse. Office départemental agricole de la Corse, Marseille, $44 \mathrm{p}$. 
80. Giraud-Genet L., r922. En Corse, le relèvement économique de l'île, le problème sylvopastoral. Imp. Samat, Marseille $53 \mathrm{p}$.

81. SAjous P., Boyer L., I922. L'amélioration de la race ovine corse. Marseille, 44 p.

$$
1923
$$

82. Cadore't H., 1923. La race bovine Taventaise en Corse. Bull. Soc. Agric., Savoie (39-40).

83. FAUCher D., r923. L'évolution industrielle de l'élevage ovin en Corse. Rev. Géogr. alp., $277-283$.

84. Laurent, 1923. L'agriculture Corse. Bull. Off. Reg. Midi 1922 et I923. C.R. in Rev. Géogr. alp., I923, 657 .

85. Vilitat L., 1925. La Corse de 1768 à 1789. Thèse - Besançon.

$$
\text { I } 926
$$

86. Joleaud I., I926. Les mammifères. Histoire du peuplement de la Corse. Bull. Soc. Sc. Hist. nat. Corse, 45.

87. Boyer M., Sajous M., I929. La race de la Corse. Congrès du mouton, Paris, 2, II 5-I 35.

88. Maugini A., I929. Problemi agricoli della Corsica. L'Italia A gricola, 629-638.

$$
\text { I93I }
$$

89. Comitr S., r93r-1932. La Corse du Sud : essai de géographie physique et humaine Bull. Soc. Sci. hist. Nat., Corse, 51-52 (502-513), I-345.

9o. Natal, J. B., I931. Parmi le thym et la rosée, chez les bergers du Cuscio (Corse). Revue de la Corse, 12, 49-57, 97-105, I45-157, 206-220, 251-257.

$$
\text { I932 }
$$

91. GIESE W. voN, I932. Die volkstümliche Kultur des Niolo (Korsika). Wort. Säch., 14, I $09-145$.

92. Gaudot G., 1932. L'exploitation du mouton en Corse. J. Agric. prat., Paris, 377-380.

93. Natali J. B., r932. Parmi le thym et la rosée, chez les bergers du Cuscio (Corse). Revue de la Corse, 13, 24-35, 77-87, I05-1 I 4, I 70-184, 2 I I-222, 249-266.

94. LE Petir BASTiaIS, I932. La production agricole de la Corse en r93o. Le Petit Bastiais, Bastia 22, 23, 24/2/1932.

95. VATti G., I932. Aspetti sardo-corsi del problema zootecnico. in Corsica Antica e moderna, Livorno, 223-230.

\section{I933}

96. Albitreccia A., 1933. Situation économique de la Corse. Bull. Ass. Géogr. fr.

97. Morandiere (C. DE LA) i933. Au cour de la Corse : le Niolo. E. Desfossés, Paris, 268 p.

98. Natali J. B., 1933. Parmi le thym et la rosée, chez les bergers du cuscio (en Corse). Revue de la Corse, 14, I 26-134, I 76-191.

99. Rosari J., I933. L'agriculture en Corse au siècle dernier. Le Petit Marseillais, Marseille, Ed. de la Corse, $17 / 2 / 33$.

$$
1936
$$

roo. Carlotri J., 1936. Monographie agricole de la Corse. Bull. Soc. dép. encourag. agric., Bastia. Rés. in Ann. Géogr., Paris, 1937, 625-628.

ior. Carlotti J., I 936. Monographie agricole de la Corse. Éditions de la Corse agricole, Ajaccio, $320 \mathrm{p}$. 
I937

IO2. MUSÉE NATIONAL DES ATP, 1937. Enquête sur l'ancienne Agriculture Corse.

io3. Carlottr J., 1939. L'élevage en Corse. Union ovine, II, 49-53.

I94I

104. BorTolan M., 194I. Potenziamento zootecnico in Corsica. in Corsica, numero unico di Azione irredentista corsa, Costelli, Milano, XIX, 29-3I.

\section{942}

105. AlBITRECcia A., I942. Le plan terrier de la Corse au XVIII e siècle. Thèse Paris $280 \mathrm{p}$. I06. AlbiTreccia A., I942. La Corse et son évolution au XIXe et au début du XXe $\mathrm{XX}^{\mathrm{e}}$ siècle. PUF, Paris, 296 p.

$$
\text { I } 946
$$

107. Pieretrit A., 1946. Les formes d'exploitation et de peuplement d'une plaine méditerranéenne : la plaine orientale de la Corse. Bull. Soc. Géogr. Marseille, 43, 7-20.

io8. TRanti N., 1946. Le berger corse. Costa, Bastia, 20 p.

$$
\text { I } 948
$$

I09. Franceschini J., I948. Les phénomènes de transhumance dans le bassin du Taravo. D.E.S. Aix-en-Provence.

Iro. LeRoy A. M., r948. Le mouton. Hachette, Paris, 46.

$$
\text { I950 }
$$

II I. C.T.C.E.P., I950. Plan de mise en valeur de la Corse, inventaire 1949. C.T.C.E.P.

$$
\text { I95I }
$$

I I2. PiERETrir A., I95I. En Corse, le désert des Agriates. Contribution à l'étude des rapports de l'homme et du sol dans les tégions méditerranéennes. Rev. Géogr. Lyon, 26, I55-I64.

\section{I952}

II3. Dumont R., 1952. Une agriculture méditerranéenne traditionnelle : la Corse. Rev. Min. A gric. (77), 237-243

$$
\text { I953 }
$$

i 4. Dumont R., i953. L'agriculture Sarde : un exemple pour la Corse. Commissariat général au plan.

I 15. Dumont R., 1953. Insuffisance et possibilités de l'agriculture Corse. Commissariat général au plan, $29 \mathrm{p}$.

i 6 . Jeannin A., I953. L'enquête pastorale de 1949-50 et la question pastorale en Corse. Féd. fr. Econ. alp., Congrès de 1953, 45-86. 


\section{I954}

I17. Jeannin B., 1954. La question pastorale corse. Rev. Géogr. Lyon, 2, I3I-I34.

\section{I955}

I 18. Degos G., 1955. Un équilibre sylvo-pastoral est-il possible en Corse? Bull. Féd. fr. Econ. alpestre.

I 19. ETTORI F., I955. Inféodation et mise en culture des plaines corses aux XVIe et XVII siècles. Etudes Corsés (6), 46-6o.

120. Hudault C., 1955. Les possibilités de l'Agriculture corse. Bull. techn. Inf. Serv. agric. (99), 3OI-3I 4 .

I21. LEFEBVRE P., 1955. Population urbaine et population rurale en Corse. Études Corses $(7,8), 86-97$.

122. Programme D'ACtion RÉgIONALE DE LA CORSE, I957. J. off. Rép. franç. I9-4-1957, 15.

\section{I956}

123. Degos G., 1956. L'élevage ovin en Corse. Bull. Féd. fr. Econ. alpestre 6, 155-175.

I24. Degos G., 1956. Le maquis, pâturage corse. Bull. Féd. fr. Econ. alp. 7, 226.

125. LAMOTTE P., I956. La structure sociale d'une communauté de la Rocca : Fozzano. Études corses, (II), 35-47.

I26. LAMOTTE P., 1956. Le système des "prese" et les assolements collectifs. Études Corses (10), 54-59.

I27. VIANGALLI [Jeanne, I956. Les structures agraires corses. D.E.S., Aix-en-Provence et Études corses (15-16).

\section{I957}

I28. ETTORI F., I957. La mise en valeur agricole de la corse au XVII' siècle. Études Corses, (I5-16), 29-47.

I29. LAMOTTE P., I957. Restrictions du droit de la propriété et sauvegarde des intérêts de la collectivité dans 1'ancienne Corse. Études corses (I5-16).

130. Menasseyre R., I957. La mise en valeur de la Corse. Rev. Min. agr. Paris (1 24), I86-ig6.

I3r. MeYer-Heine G., I957. Premiers éléments pour une étude d'aménagement de la Corse. Direction de l'Aménagement du Territoire, Paris.

132. Simi P., i 957 . Les aspects régionaux de la Corse : le Nebbio. Rev. Géogr. alp. 45, 7 I I-762,

$$
\text { I } 958
$$

133. Chiva I., 1958. Causes sociologiques du sous-développement agricole : l'exemple đe la Corse. Cahiers internationaux de Sociologie (24) I-6.

I34. Hercher P., I958. Étude d'économie agricole sur la Corse. Somivac, Bastia, 2 tomes : $80 \mathrm{p}$ et $22 \mathrm{p}$.

\section{I959}

135. KeLLER M., I959. Le plateau pastoral du Niolo. Bull. Féd. fr. Econ. alp. (9) (1958-59), 49-52.

136. KELLER M., I959. Le troupeau pépinière de Casabianda. Bull. Féd. fr. Econ. alp. (9), $63-65$.

I37. Molinier R., I959. Étude des groupements végétaux terrestres du Cap Corse Bull. Mus. Hist. nat. Marseille (3r).

r38. PANIS M., 1959. La région du Coscione. Bull. Féd. fr. Econ. alp. (9) 49-52.

139. TESTANIER J., 1959. Étude des actions en montagne, une région typique : le Niolo.

$$
\text { I960 }
$$

I40. ARmani V., I96o. La vie pastorale du Niolo. ATP, 62-34. 3 p. dactyl.

141. Documentration Française, 1960. La Corse 1960. (Monographie agricole départementale). Documentation française, Paris. 
142. Deggos M., 1960-6r. Une région typique, le Niolo. Bull. Féd. Écon. Mont. (II).

I43. SpInosi F., I960. Essai sur l'économie rurale Corse au XVII et XVIIIe. Thèse de droit, Aix-en-Provence, 405 p.

\section{I96I}

I44. Gensoliten J. P., I96r. L'exploitation pastorale en Corse. G.R. de la Corse, 9 p., ronéoté.

I45. NeUwirTh M., r96r. Rapport sur la situation économique de la Corse et sur les travaux d'aménagement agricole et touristique. Imp. Assemblée Nationale Paris (1322).

146. Otravi P., I96r. Une expérience sur la productivité laitière de la brebis Corse. Patre $(87)$, I0-1 I.

147. REPARAZ (G. A. DE), I96r. L'évolution des structures agraires dans quelques communes typiques de la Corse. Bull. Soc. Sci. hist. nat., Corse. (559), 3 I-53 (560), 5 I-75.

148. S.o.m.r.v.A.C., I96r. Mise en valeur agricole et touristique de la Corse, Paris, 32 p.

$$
\text { I } 962
$$

I49. Jfan-Bruhnes Dei,amarre, Mariel et al., I962. Bergers de France. Catalogue de 1'exposition au Musée des Arts et Traditions populaires, Paris, 6/7, 19/1 1/1962. Arts Trad. pop., 10, I-328.

150. KOLODNY Y., I962. I'utilisation des sols en Corse : sur la décadence d'un terroir insulaire. Union Géographique internationale, Commission des terres ariđes, Grèce, r962 (cité par SIMI, I966).

151. Lucrani, 1962. L'identification des brebis Corses. Patre (99), I8-20.

I52. Pomponi Janine, r962. La vie rurale de deux communes corses : Serra di Scopamene et Sotta. Mém. maîtrise, Géographie humaine, Aix-en-Provence, La Pensée universitaire, Aix-en-Provence.

I 53. REPARAz (G. A. DE), I962. Diversité et évolution des structures agraires Corses. Méditerranée (I) 55-56.

I54. Sim P., I962. L'aménagement des secteurs ruraux de la Corse. Brochure du Génie Rural, Bastia, ro5-111.

I55. S.O.M.I.V.A.C, I962. Le programme d'équipement et de mise en valeur agricole de la Corse dans le cadre des grands aménagements régionaux. S.O.M.I.V.A.C., Bastia, 33 p.

$$
\text { I } 963
$$

I 56. BAticle Y., I963. L'évolution de l'élevage des ovins et des caprins dans les pays méditerranéens. Ann. Géogv., I, 99-roo.

I57. Carlotri J., 1963. La brebis et le berger dans leurs rapports avec la forêt et la vie en haute vallée. Demain la Corse, janv. 8-I4, fév. 8-r 2.

I 58. ChIVA I., I963. Social organisation, traditional economy and customary law in Corsica. In PITT-RIVERS, Juiran, Mediterranean countryman, Mouton, Paris, La Haye, 97-I 2.

I59. D.D.A. DE LA CoRSE, I963. Journée de l'Élevage. D.D.A. de la Corse.

I60. DEGos G. 1963. Rapport à la Société Corse d'Économie sylvo-pastorale de mai r963. Cité par Simi.

I6r. Dupias G., r963. Notice sommaire de la carte de la végétation de la Corse C.N.R.S. Paris.

I62. LAMOTTE P., I963. En hommage à Vincent ARMANi, dessins relatifs à la vie des bergers corses. Corse hist. archéo. litt. scient., 3, 5I-55.

i63. Martini M., i963. Le désert des Agriates, vieille terre d'abondance. Bull. Soc. Sci. Hist. nat. Corse (569) 7-18.

164. Massignon Geneviève, I963. Contes corses. Orphys, Gap. 38o p.

I65. Pfeffer P., I963. Premières estimations sur le statut et la structure des populations de mouflon (Ovis musimon, Pallas) dans la réserve nationale de Bavella, Corse. Terre et Vie, 2, 129-1 39.

I66. QUiTTET E., I963. Races bovines. La Maison rustique, Paris, 36.

167. Vinciguerra S., ig63. La vie en Corse au Moyen-Age. L'Union Corse.

$$
\text { r964 }
$$

i68. Bevilacqua M. V., I964. Organisation de la vie rurale dans la basse vallée du Porto. D.E.S. Géographie, Aix-en-Provence.

I69. Carlót'ti J., i964. L’élevage ovin en Corse. D.D.A. Corse, Ajaccio, i i p. ronéoté. 
I 70. D.D.A. DE LA CORSE, 1964. La prairie temporaire en région sèche.

I7I. Romani, r964. Le problème de la viande en Corse (Journée de l'Élevage, Corte, 1964). S.O.M.I.V.A.C., SETCO. (27) 7-13.

I72. Rondeau A., r964. La Corse. Armand Colin, Paris, 94-97, I I4-I 20.

I73. S.O.M.I.V.A.C., I 964 . L'élevage bovin en Corse. Bastia, 62 p. ronéoté.

\section{965}

I74. Chabaud A., I965. Mouflons de Corse et d'ailleurs. Bêtes et nature, 19, 43-47.

175. D.D.A. DE LA CORSE, r 965 . Enquête préliminaire à la préparation du Ve plan. Note sur la population et les structures agricoles de la Corse. Ajaccio, ronéoté

I76. D.D.A. DE LA CORSE, I965. Évolution de la population active agricole au cours de la période du Ve plan. Ajaccio.

I77. D.D.A. DE LA CORSE, I965. La population agricole de la Corse après l'enquête par sondage de r963. Ajaccio.

I78. DELOGU M. R., I965. La production de l'agneau de boucherie en Corse. S.O.M.I.V.A.C.SETCO (34) 23-26.

I79. Dupias G., Gaussen H., Izard M., Rey P., i 965 . Carte de la végétation de la Corse au I $/ 200000$. C.N.R.S. Paris.

I 80. MAISANI M., I 965 . Groupements agricoles d'exploitation en commun. D.D.A. de la Corse.

181. Massignon, Geneviève, 1965. Vie pastorale en Corse. Rev. Ling. Rom., (I I 5-1 16), $209-227$.

182. PaoretTi, 1965. De quelques aspects de l'élevage caprin en Corse. D.D.A., Ajaccio 5 p., ronéoté.

I83. QUiTTET E., I965. Races ovines françaises. La Maison rustique, Paris, 46-47.

184. Ravis-Giondani G., 1975. L'univers dans une omoplate. Bull. Soc. Sci. hist. nat. Corse, 6I5-6I6 et in BALFET, et al., I976. Pratiques et représentations de l'espace dans les communautés méditerranéennes. C.N.R.S., Paris.

I 85. S.O.M.I.V.A.C., I965. Marché d'économie agricole no ${ }^{\circ}$. Étude de détail des Plaines de la Balagne. Mémoire général. Service du Génie Rural, Ajaccio, I22 p.

\section{966}

I86. LE Mouton, I966. Une industrie pastorale : le Roquefort. I. - La production du lait de brebis. II. - La production du fromage. Le Mouton, 89-90, I I I-I 2.

187. Barroli A., I966. L'aménagement du territoire et le problème Corse. Mém. Inst. Ett. polit., Aix-en-Provence.

I 88. CERUTTI J., I966. L'élevage ovin en Balagne. S.O.M.I.V.A.C.-SETCO (36), 10-19.

I89. ClaReT R., ig66. L'élevage ovin et la production fromagère en Corse. Thèse Vét. Alfort, $92 \mathrm{p}$.

190. Nizza P. F., 1966. Some characteristics of the Corsican Swine, in BuSTAD L. K. et al.: Swine in Biomedical research, Proc. Symp. Richland, Washington, 775-779.

i9i. Perfetitini M., ig66. Vie et évolution d'une communauté rurale de la montagne Corse. D.E.S. Géographie, Aix-en-Provence.

I92. Silvagnola A., I966. L'homme et le milieu naturel de la vallée d'Asco. Bull. Soc. Sci. hist. nat., Corse (58I), 49-97.

I93. Srmi P., I966. L'adaptation humaine dans la dépression centrale de la Corse. Thèse Géographie humaine, Aix-en-Provence, Orphys, Gap, 260 p.

I94. WeIss M. C., I966. L'évolution des bergers de Gradule. La Corse historique, 6 (2 I-22), $3 \mathbf{I}-36$.

$$
\text { I } 967
$$

I95. BARRÉ G., I967. La machine à traire les brebis. S.O.M.I.V.A.C.SETCO (4I), $24-28$. 196. CERUTTi H., 1967. Les cultures fourragères en Corse. S.O.M.I.V.A.C.-SETCO (41) I 3-19. I97. D.D.A. DE LA CORSE, 1967. Enquête porcine en Corse. Ajaccio,

198. Figaro Agricol,E, 1967. En I957 l'Agriculture n'était plus qu'un souvenir. Figaro Agricole, Oct. I967, Paris.

199. F.N.S.E.A., I967. La Provence et la Corse agricole. Connaissance de l'Agriculture (10), IO-II.

200. HERChER P., I967. En matière d'introduction (I). S.O.M.I.V.A.C.SETCO (4I), I 2.

(I) A une journée sur les fourrages. 
201. MASON I. L., 1967. Sheep breeds of the Mediterranean. FAO, Rome, 73-74.

202. Pfeffer P., 1967. Le Mouflon de Corse (Ovis ammon musimon, Schreber, I 782); Position systématique, écologie et éthologie comparée. Mammalia, 31 (suppl.), 262 p.

203. Sarre M., Palmieri M., I967. L'aviculture ultra-moderne en Corse. L'électricité ä la terme (2I), I2-I3.

204. WIL,D M., 1967. Un atout sérieux : l'ensilage. S.O.M.I.V.A.C.-SETCO (4I), 20-24.

$$
\text { I } 968
$$

205. Dajoz R., 1968. Le moufion de Corse menacé chez lui de disparaître. Science Progrès, La Nature (3 399), 249-252.

206. D.D.A. DE LA CORSE, I 968 . Enquête communautaire sur la structure des exploitations agricoles en I967. Ajaccio.

207. D.D.A. DE LA Corse, 1968. Enquête porcine en Corse. Ajaccio.

208. MiQUeT J., I968. Quelques aspects de l'élevage caprin en Corse. La chèvre (50), I 8-22.

209. TuUssarnt G., I968. L'élevage caprin en Corse, un essai de modernisation à Favone. S.O.M.I.V.A.C.-SETCO (47) $3 \mathrm{I}-37$.

2 го. Toussaint G., r968. Étude sur l'élevage caprin en Corse. La technique laitière (60o), I 5-I 7 .

$$
\text { I } 969
$$

2ri. Aumaitre A., 1969. Compte rendu de mission (porcins) en Corse. C.N.R.Z. - I.N.R.A. Jouy-en-Josas.

212. D.D.A. DE LA Corse, 1969. Enquête porcine en Corse. D.D.A. Ajaccio.

21 3. Flaman'T J. C., I969. Rapport à la Direction de la S.O.M.I.V.A.C. sur l'installation d'une Station d'élevage de brebis laitières Corses. I.N.R.A. - C.N.R.Z., Jouy-en-Josas. 9 p. dactyl.

2 i 4. Praloran J. C., Ecochard M., Goto N., ig69. Développement et Amélioration de l'Agriculture en Corse. Ministère de l'Agriculture, Direction générale de l'Esspace rural.

\section{970}

215. ArRighi P., 1970. Isa vie quotidienne en Corse au XVIIIe siècle. Hachette, Paris, 20-2 I, I67-17I.

2i6. CotTe M. A., MicalfFF P., LETELLIER, I97o. Rapport de mission en Corse (sur l'amélioration fourragère). I.N.R.A., Paris.

2I 7. D.D.A. DE LA CoRSE, 1970. Enquête porcine en Corse. D.D.A., Ajaccio.

218. Galiani Jeanne, r970. Démographie et économie d'une cellule Corse : Coti Chiavari. Mémoive de Maîtrise, Lyon.

219. GARDELLE C., 1970. Impressions pastorales corses, été 1969. Rev. Géogr. alp. (2).

220. Guiard-Marigny Hélène, r970. L'élevage ovin dans l'économie rurale Corse. Thèse Vét., Lyon, $59 \mathrm{p}$.

221. Meria G., 1970. La Castagniccia, terre d'exode ou terre d'avenir? Bull. Soc. Sci. hit. nat. Corse (594), 23-87.

222. Pichod-Viale D., Denise, i97o. Protection de la faune sauvage corse. Bull. Soc. Sci. hist. nat., Corse (597), 3-36.

223. RenuCCI Janine, I97o. L'élevage en Corse, un archaîsme menacé. Rev. Géogr., Lyon. $357-389$.

$$
\text { I97I }
$$

224. C.P.S., I97I. Recueil d'informations sur l'élevage extensif du porcen Corse. C.P.S., Corte, 17 p. ronéoté.

225. CERUtTI J., 1971. La culture de l'herbe et son exploitation. S.O.M.I.V.A.C.-SETCO (59) I 9-26.

226. Colombani F., i97I. L'élevage ovin en Corse. D.E.S. Géographie, Aix-en-Provence.

227. D.D.A. DE LA CORSE, I97I. L'économie agricole dans l'espace rural de la région Corse. Bull. Féd. Econ. mont. Grenoble.

228. D.D.A. DE LA CORSE, I97I. Enquête porcine en Corse. D.D.A. Ajaccio.

229. D.S.V. DE LA CORSE, I97I. Liste des Syndicats d'élevage de la Corse. D.S.V., Ajaccio.

230. Falcucci C., I97I. La vie des communautés agraires de la piève de Casinca aux XVII ${ }^{\circ}$ et XVIIIe. Mémoire de Maîtrise d'histoire, Nice, $207 \mathrm{p}$. 
23I. LEenhardT M., I97I. L'élevage ovin. Courrier du Parc de la Corse (3), 17-20.

232. MicalefF P., I97I. Réflexions sur la production fourragère en Corse. S.O.M.I.V.A.C., SETCO (59), I6-I8.

233. Perret G., Peyraud D., Lucien C., I971. La race Corse, in ITOVIC, Les races ovines françaises, séries de brochures, COFRANIMEX Paris, $6 \mathrm{p}$.

234. QUITTET E., ZERT P., I97I. Races porcines en France. La Maison Rustique, Paris, 37-38-

235. RENUCCI, Janine, I97I. L'élevage en Corse un archaïsme menacé (extrait de thèse). S.O.M.I.V.A.C.-SETCO (58), $17-2 \mathrm{I}$.

\section{972}

236. Albertini J. P., I972. La vie des communautés de la piève de Luri aux XVIIe et XVIIIe siècles. Mémoire de Maîtrise d'Histoire, Nice I $46 \mathrm{p}$.

237. AUjourd'hui la CorSE, I972. Modes de conduite et importance du cheptel caprin dans le département. Aujourd'hui la Corse, 3 oct. Io-II.

238. Blasini P., I972. Le Niolo, étude d'aménagement rural. D.D.A. Région Corse - I92 p + annexes.

239. CerutTi J., 1972. Pour le S.O.M.I.V.A.C. un impératif : aider à la rénovation de l'élevage en Corse. S.O.M.I.V.A.C.-SETCO (62), I 7-22.

240. D.A.T.A.R., I972. Schéma d'aménagement de la Corse. Travaux et recherches de Prospectives $n^{\circ} 32$.

24I. D.D.A. DE LA CORSE, I972. Critères de reconnaissance des groupements de producteurs, élevage porcin et viande porcine. Ajaccio.

242. LALEURE J. P., I972. La montagne corse : les hautes vallées du Prunelli et de la Gravone. Mémoire de Maîtrise Géographie, Aix-en-Provence, I45 p.

243. Lejeal P., i972. La paix des brebis. Courrier du Parc de la Corse (9), I3-I 5.

244. Paoletti M., Maniccia P., I972. Recensement général de l'Agriculture 1970-197I; Les exploitations agricoles - Premiers résultats. S.C.E.E.S., D.D.A. de la Corse, S.S.D.

245. Praloran J. C., I972. L'élevage porcin dans les zones de montagne de la Corse. D.D.A. Ajaccio, I63 p. ronéoté.

246. RAvis-GIORDANI G., I 972. Errance du troupeau, errance du berger. CERESM, Université, de Provence, $I 7+2$ p. ronéoté.

247. S.C.E.E.S., 1972. Recensement général de l'agriculture I970. Enquête communale, 20, Corse. S.C.E.E.S. Paris, 139 p.

248. S.C.E.E.S., 1972. Recensement général de 1'Agriculture 1970-1971. Fascicules départementaux 20, Corse. S.C.E.E.S., Paris. Imprimerie nationale, $246 \mathrm{p}$.

249. S.C.E.E.S., D.D.A. DE LA CORSE, I972. Recensement général de l'agriculture I970-7I. 20, Corse. Inventaires communaux - premiers résultats. S.C.E.E.S., D.D.A. Ajaccio.

250. VIvas J. P., 1972. Structures agraires et vie paysanne dans un village corse : Castifao au XIXe siècle. Mémoire de maîtrise, Aix-en-Provence.

I973

251. Anonyme, 1973. Analyse du mileu rural Corse. $76+$ XXIII p. ronéoté.

252. Casabianca F. DE, Flamant J. C., Molénat G., 1973. Rapport sur les possibilités d'une action " élevage " dans le cadre du programme D.G.R.S.T. "Maquis Corse ". ronéoté s $8-20 / 9 / 73,3 \mathrm{p}$.

253. ConRad Marcelle, I973. Les incendies en Corse : aperçu de leurs multiples conséquences. Bull. Sci. S. Hist. Nat. Corse (607), 43-50.

254. Conrad Marcelle, 1973. Essai d'ethnobotanique corse. Bull. Soc. Sci. hist. nat. Corse (607) $5 \mathrm{I}-63$.

255. Lanfranchi F. DE, Weiss M. Cl., I973. La civilisation des Corses - Les origines. Ajaccio, I $82 \mathrm{p}$.

256. Irenclud G., Ravis-Giordani G., 1973. Pour une ethnologie de la Corse - Etat actuel des recherches. Etudes corses, 1, I93-232.

257. MONTARD, F. DE, 1973. Pâturages en Corse. 3 p. dactylogr.

258. Nivaggioni T., 1973. Vie matérielle et économique de Bastelica aux XVII e et XVIIIe siècles. Mémoire de Maîtrise d'Histoire, Nice, $112 \mathrm{p}$.

259. S.C.E.T., O.N.F., I973. La rénovation de l'économie rurale in "Corse, Parc naturel régional A, rapport général ", S.C.E.T., O.N.F., Paris. ch. 2, 74-92. 


\section{I974}

260. Acquaviva M., 1974. Enquêtes socio-économiques dans le cadre du programme de recherches avec la D.G.R.S.T. Rapport D.G.R.S.T., Paris.

26r. A.P.E.E.M., I974. Étude écologique de la dégradation du maquis et de sa régénération dans le bassin du Fango (Filosorma). Bastia, ronéoté.

262. BATICLE X., I974. L'élevage ovin dans les pays européens de la Méditerranée occidentale. Les belles lettres, Paris et Publications de 1'Université de Dijon XLVII, 269-295.

263. BeRENni P., DeLivarRe G., LANGi.ET B., I974. Recherche des possibilités de développement agricole dans la zone du Golo, Corse. E.N.S.A., Toulouse.

264. Chambre départementale d'Agriculture, C.D.J.A., Section de développement de la F.N.S.E.A., G.V.A. et C.E.T.A., I974- Région de Corse. Programme pluriennal de développement agricole $1974-78.32+5$ p. ronéoté.

265. C.P.S., I974. Les potentialités physiques, économiques et sociales du Sartenais. . C.P.S., Corte.

266. Defrancheschi J., I974. Pasteurs et cultivateurs en Corse au XVIII e siècle. Ann. hist. Révolution tranç. (2 I 8), 542-556.

267. E.D.E. CorSE, I974. Rapport d'activité. Ajaccio, 4I p. ronéoté.

268. Exposition ARTS ET TRADITIONS POPUlaIRES DE CORSE, 1974. Catalogue. Corte, I5-I 8 Août 1974 .

269. Guérin G., I974. Contribution à l'étude écologique de la Castagniccia A.T.R.S.P., Ajaccio.

270. LECA J. C., I974. Structures agraires, démographie et vie paysanne d'une communauté Corse au XIXe. Mémoire de Maîtrise d'Histoire, Aix-en-Provence, I55 p.

271. MaGdener Evelyne, I974. Recherches sur les communautés rurales en Corse au XVIIIe siècle : la province de Vico. Aspects économiques et sociaux : $177^{0-1} 792$. Mémoire de Mâ̂trise, Paris.

272. PERNET F., 1974. Rapport scientifique annuel pour 1974. Annexe II : premières hypothèses de travail pour l'étude socio-économique du pastoralisme de l'intérieur Corse. 15 p. ronéoté.

273. Perrier E., 1974. Corse : les raisons de la colère. Économie et Politique $\mathrm{n}^{\mathrm{o}}$ hors série.

274. Pomponi F., I974. Un siècle d'histoire des biens communaux en Corse (I770-1870). Études Corses (3), 5-4I.

275. Pomponi F., I974. Les cahiers de doléances des Corses de i 73o. Bull. Soc. Sci hist. nat. Corse (6ro) 7-65.

276. Racon S., I974. Place de l'élevage caprin dans l'ensemble de l'élevage en Corse, in Étude écologique de la dégradation du maquis et de sa regénération dans le bassin du Fango (Filosorma). Rapport des activités menées jusqu'en 1974. A.P.E.E.M., ronéoté.

277. Renuccr Janine, 1974. Corse traditionnelle et Corse nouvelle. Audin, Lyon, 203-233.

278. Simi P., I974. Le rôle géographique du maquis. S.O.M.I.V.A.C.-SETCO (7I) 39*47 (72), 43-48.

279. S.R.S.A., 1974. L'élevage ovin Corse en I974. S.R.S.A.

\section{I975}

280. Alivi (L'), I975. Plan d'aménagement rural du territoire communal de Sollacaro. Projet d'orientation. Association de Rénovation rurale de la basse vallée du Taravo.

28r. Alsmark G., 1975. Korsikanske hyrder (Bergers Corses) (en suédois). Bygd, 6 (3) 7-25.

282. ANONYME, I 975 . Évolution de la production fruitière du maquis, mesures de productivité effectuées dans la vallée du Fango. C.P.S., A.D.I.R., A.P.E.E.M. no spec. I. III.

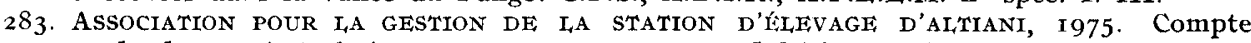
rendu des essais techniques, campagne 1974-1975. S.O.M.I.V.A.C., Bastia, 83 p.

284. BARRy J. P., MANIÈRE R., I975. Histoire et végétation d'une commune rurale corse de la vallée du Tavignano. Université de Nice.

285. B.D.P.A., r975. Carte de l'utilisation du sol et de l'occupation des terres : la Corse I/I00 ooo. M.J.P.A.E.N.M.

286. BLASINI P., I975. Le Cap Corse - Étude d'aménagement 1973-1974. Région Corse et D.D.A., Ajaccio.

287. Bourgeat F., 1975. Cartes des unités édaphiques et géomorphologiques en vue de l'aménagement de la vallée du Golo, I/50 ooo. E.N.S.A. Toulouse.

288. Charpentié B., Berenni P., I975. Étude socio-économique préalable à la constitution d'associations foncières pastorales en Corse. E.N.S.A., Toulouse.

289. ColonNa J. N., 1975. Roquefort d'accord. Kym, I975 (52), 37-38. 
290. Conrad Marcelle, 1975. Les plantes sauvages dans la vie quotidienne des Corses. Bull. Soc. Sci. hist. nat., Corse (6r4), 61-75.

291. D.D.A. DE LA CORSE, I975. L'élevage ovin Corse en I974. Ajaccio, 8 p. ronéoté.

292. ETIENNE M., I975. Étude d'améliorations pastorales dans la vallée du Golo, Corse, premiers bilans. M.I.P.A.E.N.M. - S.E.E.E.E.

293. ETIENNE M., I975. Etude d'améliorations pastorales dans la vallée du Golo, Corse. Premier rapport partiel. M.I.P.A.E.N.M., 77 p. ronéoté.

294. ETIENNE M., I975. Étude d'améliorations pastorales dans la vallée du Golo, Corse. Deuxième rapport partiel. M.I.P.A.E.N.M., 37 p. ronéoté.

295. ETIENNE M., I975. Étude d'une amélioration pastorale dans la vallée du Golo. S.E.E.E., C.E.P.E., Montpellier, ronéoté.

296. Fédération Ovine DE Corse, 1975. Propositions pour un aménagement de l'élevage en Corse, ronéoté.

297. Flamant J. C., I975. Aide-mémoire sur le développement des recherches zootechniques en Corse. S.A.G.A., I.N.R.A., Toulouse. ro p. ronéoté.

298. Flamant J. C., I975. Motivations d'une action concertée zootechnie $\times$ phytosociologie et écologie $\times$ sciences humaines dans le bassin méditerranéen. 2 p. ronéoté.

299. FlamanT J. C., I975. Réflexions et propositions sur la réalisation de recherches sur l'élevage en Corse. S.A.G.A., I.N.R.A., Toulouse, 9 p. ronéoté.

30o. Geri C., LENCLUD G., I975. Etude des conditions agro-techniques du développement de l'élevage dans une zone pilote de la montagne corse. I.N.R.A., I, a Minière et Univ. Paris Nanterre.

301. LANFranchi F. DE, Weiss M. C., 1975. La civilisation des Corses - Les peuplades de l'âge du fer, Ajaccio, 2I4 p.

302. LAURANS R., I975. Chiens de garde et chiens de conduite des moutons. Ethnozootechnie (I2), I5-I8.

303. LAURANS R., 1975. Les sonnailles et leur relation avec la transhumance en Corse et en Sardaigne. Ethnozootechnie (n ${ }^{\circ}$ spécial, 2 Quelques Aspects de la Transhumance), 59-66.

304. LaUvergne J. J., 1975. Situation ethnique comparée de la Corse et de la Sardaigne en 1974 : bovins, ovins, caprins, porcins et équidés. In " Etude pilote sur la conservation des ressources génétiques animales ". F.A.O., Rome, Annexe no 1 , I 7-24.

305. ML/MTB, 1975. Présentation du Roquefort Maria Grimal. Le 5 mai $1975,4+2$ p. ronéoté.

306. Pirti-Ferrandi A., I975. Étude préliminaire à un programme de relance de l'élevage ovin laitier Corse. Mém. fin d'études E.S.I.T.P.A., Paris. 42 p. + annexes.

307. PoLI Gisèle, 1975. Le temps des nouveaux chevriers. Kyrn (3), 35-39.

308. Pomponi F., i 975 . Un siècle d'histoire de biens communaux en Corse, i770-1870. Études corses (5), 15-54.

309. PRÉFECTURE DE CORSE, I975. Une région française : la Corse.

310. Ravis-GroRdani G., I975. Le troupeau errant, l'utilisation de l'espace par les bergers du Niolu (Corse). In Ethnologie et Editions sociales, Paris, 285-301. S.O.M.I.C.A.V.SETCO, 1975 (76), 39-45 et Fourrages, 1975 (63), 3-21.

3Ir. S.C.E.E.S. et D.D.A. d'Ajaccio, 1975. 'L'élevage ovin corse en 1974 (résultat d'une enquête par sondage). Ministère de l'Agriculture, $8 \mathrm{p}$. ronéoté.

312. S.E.I., 1975. Premier bilan des études entreprises dans le massif de la Castagniccia (Corse). I.N.R.A., S.E.I., Versailles, ronéoté 79 p.

313. S.U.A.D., I975. Propositions pour une politique de l'élevage. Chambre d'Agriculture de la Corse, 20 p. ronéoté.

314. VERNEUIL, B. DE, I975. Essai de comportement de quelques fourrages en Castagniccia. I.N.R.A. - S.E.I., Versailles.

315. VIALE, Denise, r975. Essai d'appréciation des ressources alimentaires réelles du maquis. C.P.S. Corse, A.D.I.R. et. AP.E.E.M., No Spéc. déc. 1975, I-10.

\section{976}

3i6. Association pour la gestion de la station d'élevage d'Altiani, i976. Compte rendu des essais techniques. S.O.M.I.V.A.C., Bastia.

317. BARRY J. P., MANIÈRE R., I976. Approche de la phyto-dyriamique méditerranéenne d'après la végétation d'une commune de la Corse : Antisanti. Univ. Nice.

318. B.D.P.A., S.O.D.E.T.E.G., 1976. Carte des caractéristiques du couvert végétal de la Corse $1 / 25$ ooo. M.I.P.A.E.N.M. 
319. BRUGieRE D., 1976. Quelques chiffres globaux sur la Castagniccia. S.E.I., C.N.R.A., Versailles.

320. Conrad, Marcelle, r976. Le maquis du Filosorma et essai d'ethnobotanique. Bull. Soc. Sci. hist. nat. Corse (625), 19-25.

321. ConRad Marcelle, 1976. Les espèces végétales du maquis Corse. S.O.M.I.V.A.C.-SETCO, (77).

322. ExiEnNe M., I976. Cartes de potentialités pastorales de la région du Golo Corse $1 / 25$ ooo. M.I.P.A.E.N.M., S.O.D.E.T.E.G.

323. ETIENnE M., 1976. Carte de l'occupation des terres de la région du Golo-Corse $1 / 25$ ooo. M.I.P.A.E.N.M., S.O.D.E.T.E.G.

324. FriENNE M., 1976. Études d'améliorations pastorales dans la vallée du Golo Corse. $2^{\mathrm{e}}$ rapport partiel. M.I.P.A.E.N.M., S.E.E.E.E.

325. ETrENNE M., I976. Éléments pour la prévention des incendies et pour l'amélioration des ressources pastorales dans la région du Golo, Corse. M.I.P.A.E.N.M., S.E.E.E.E.

326. ETIENNE M., 1976. Étude sur les conditions d'amélioration des parcours dans la vallée du Golo. In $2^{\mathrm{e}}$ Réunion du groupe d'étude des herbages méditerranéens de la F.A.O. (2023 avril 1976), II p., ronéoté, M.I.P.A.E.N.M.

327. ETIENNE M., I976. Études pour une expérimentation préalable à une mise en valeur sylvo-pastorale de l'espace naturel de Corse. S.R.A.F., Ajaccio, S.O.D.E.T.E.G.

328. Gamisans J., 1976. Projet d'étude pour l'aménagement pastoral et sylvatique des montagnes corses. Univ. Marseille.

329. GERI C., I976. Études des équilibres sylvo-pastoraux dans l'étage montagnard corse. I.N.R.A. La Minière.

330. Gindro J. C., 1976. Ucciani : un village corse de la fin du XVIII à la fin du XIXe siècle. Mémoire de Maîtrise d'Histoire, Aix-en-Provence, $236 \mathrm{p}$.

331. Gurlaine J., 1976. Premiers bergers et paysans de l'occident méditerranéen. Mouton, Paris, La Haye.

332. Lacoste A., 1976. Étude sur le maquis Corse dans le bassin du Fango. Université de Nice.

333. Lauvergne J. J., I976. Pour une meilleure typologie raciale des populations domestiques méditerranéennes. Conf. Scient. MAB Méditerranée. Montpellier 27/9-2/10, $76-4 \mathrm{p}$.

334. LaUvergne J. J., Adalsteinsson S., i976. Genes pour la couleur de la toison de la brebis Corse. Ann. Génét. Sél. anim., 8 I53-172.

335. LENCLUd G., 1976. Contribution ethnologique à l'étude des équilibres sylvo-pastoraux dans la montagne Corse (Niolo). Université de Paris.

336. LENGLUd G., PERNET, 1976. État des recherches de sciences sociales sur le pastoralisme corse. Université de Paris et Université de Grenoble.

337. LUCKE H., I976. Interprétation cartographique de l'évolution de l'utilisation du sol dans la commune de Vescovato. Bull. Soc. Sci. hist. nat. Corse (618), 67-82.

338. M.I.P.A.E.N.M., 1976. Éléments pour la prévention des incendies et pour l'amélioration des ressources pastorales dans la région du Golo, Corse. C.E.P.E., C.N.R.S., Montpellier et S.E.E.E., 78530 Buc.

339. M.I.P.A.E.N.M., I976. Étude zootechnique et expérimentation pastorale dans la vallée, du Golo, Corse. $2^{\text {e }}$ rapport partiel : campagne de mesure de l'automne 1975. S.E.E.E.E. C.E.P.E.

340. MOLÉNAT M., VeRneutr (B. DE), 1976. Réflexions sur l'élevage porcin en Corse. I.N.R.A. : S.E.I., San Giuliano Corse et C.N.R.Z. Jouy-en-Josas, Document de travail à diffusion restreinte, $64 \mathrm{p}$. ronéoté.

34I. OGIER J., 1976. Étude zootechnique et expérimentation pastorale dans la vallée du Golo corse. $3^{\text {e }}$ rapport partiel et rapport de synthèse M.I.P.A.E.N.M., S.E.E.E.E.

342. Pomponi F., I976. Problèmes de la vaine pâture en Corse au XIX e siècle. Bull. Soc. Sci. hist. nat. Corse (62I), 19-42.

343. Racon S., 1976. Le Filosorma, Structures économiques. Bull. Soc. Sci. hist. nat. Corse (625), 83-92.

344. S.E.I., I976. Premier bilan des études entreprises dans le massif de la Castagniccia, Corse. I.N.R.A., S.E.I. Versailles, 83 p. ronéoté.

345. S.E.I., I976. Présentation schématique des recherches en cours sur l'élevage en Castagniccia. C.N.R.A., Versailles.

346. S.O.M.I.V.A.C., 1976. L'élevage caprin en Corse. Bastia, 62 p. ronéoté + annexe.

347. VIALE Denise, 1976. L'utilisation de la végétation par les communautés rurales du Filosorma. A.P.E.E.M. 
348. ALIVI (L') r977. $b$ : Compte rendu des séances de travail du 4 au 8 avril 1977. Association de rénovation rurale de la basse vallée du Taravo.

349. Alivi (L') C.E.P.E., G.R.E.S.E., I977. Mise en place d'un projet d'amélioration pas torale pour le développement intégré de la commune de Sollacaro (Corse). Rapport sur l'état d'avancement des études (pér. du I-I-77 au 30-9-77. ALIVI, C.E.P.E., G.R.E.S.E. Montpellier.

350. ANTONI D., 1977. Berger sans terre. Kyrn (78), 35.

35I. A.R.E.E.A.R., I 877 . Étude sur l'impact de l'indemnité spéciale montagne dans le département de la Corse du Sud. Ministère de l'Agriculture, Région Corse. 18 p. ronéoté.

352. Brugère D., Deffontaines J. P., Raichon C., Verneuil (B. De), i977. Réflexionssur le développement des activités agricoles à l'occasion du contrat de pays de la Castagniccia. S.E.I., C.N.R.A., 20 p. ronéoté.

353. Cabrol. I., GrF́e J. P., I977. P. Nizzi, Pepino berger Corse, une vie. Hachette, Paris, $234 \mathrm{p}$.

354. Casabianca F., 1977. Enquête sur les reproducteurs porcins Corses. E.N.S.A., Toulouse, mémoire de $3^{\mathrm{c}}$ année, I05-47 $\mathrm{p}$.

355. Casabianca F., Molfinat M., I977. Enquête sur les reproducteurs porcins Corses. I.N.R.A., diffusion restreinte, $26 \mathrm{p}$.

356. Casabianca F. DE, 1977. Chataigneraie et développement de la Corse. Réflexions à partir de la Castagniccia. Station I.N.R.A. de San Ghjulianu, I 8 p. +4 annexes $(8$ p.), S.O.M.I.V.A.C., Bastia.

357. Crespo D. G., I 977. Quelques aspects de l'amélioration des productions pastorales et fourragères en Corse. Elvar, Portugal, ronéoté, $33 \mathrm{p}$.

358. Deitahaye J., 1977. La brucellose en Corse. Pâtre, avril 1977, $31-33$.

359. ENDerdin J. P., 1977. Boisellerie du Haut-Doubs et boisellerie corse. Ethnol. Fr., N.S., 7, $361-384$.

360. ErIENNE M., 1977. Bases phyto-écologiques du développement des ressources pastorales en Corse. Thèse, Université de Montpellier, 2 ro p.

36r. Flori F., 1977. L'utilisation de la glandée. Bull. Soc. Sci. hist. nat. Corse.

362. Flori J., Casta T., Navarro D., Pietri J., 1977. Puis vient l'araire. C.R.D.P., Ajaccio, $3^{8}$ p. +12 dias.

363. G.R.E.S.E. et C.E.P.E., 1977. Potentialités de développement de la commune de Sollacaro (Corse). C.R. Table ronde de réflexion, Sollacaro 8-12/1 I/76. G.R.E.S.E./C.E.P.E., Montpellier, $36 \mathrm{p}$.

364. Lauvergne J. J., Denis B., Théret M., I977. Hybridation entre un Mouflon de Corse (Ovis amon musimon SCHREBER, 1972) et des brebis de divers génotypes : gènes pour la coloration pigmentaire. Ann. Génét. Sél. anim., 9, I51-16r.

365. LoNG G., 1977. Le projet Humimed. Rapport du Comité français du MAB, 73-75.

366. LoNG G., I977. Éléments pour une évolution critique des études de base réalisées en Corse sur incitation de la M.I.P.A.E.N.M. et propositions pour le développement et l'application de ces études. C.E.P.E., C.N.R.S., Montpellier.

367. Luciani C., 1977. La race Corse. Pâtre, No spécial, 87.

368. LUCIANI F., 1977. L'élevage caprin en Corse. S.O.M.I.V.A.C. - S.E.T.C.O. (83), 17-23-

369. M.A.B., 1977. Maquis et transformation de l'espace rural en Corse. L'homme et la Biosphère $(\mathrm{MAB}), 5^{\mathrm{e}}$ session du Conseil international de coordination Recherche environnement $n^{\circ}$ 7., Documentation française, 63-67.

370. M.I.P.A.E.N.M., 1977. Étude d'appréciation économique d'un modèle pastoral rénové. S.O.D.E.T.E.G., M.I.P.A.E.N.M.

37i. Molf́nat G., Flamant J. C., Prud'hon M., Thiaulit M., 1977. Mise en valeur par l'élevage ovin des paturages secs méditerranéens. F.E.Z., $28^{\mathrm{e}}$ réun. ann. Bruxelles, ronéoté, ro $\mathrm{p}$.

372. MOLFNAT M., VERNEULL (B. DE), I977. La charcuterie en Castagniccia. Ethnozootechnie (16), 76-86.

373. Patre, 1977 Corse. Les fromages Corses. Patre (246), I03-104.

374. Pernetr-Lenclud, 1977. Berger en Corse. Essai sur la question pastorale. Presses universitaires de Grenoble, $192 \mathrm{p}$.

375. Racon S., 1977. Le Filosorma : structures économiques. Bull. Soc. Sci. hist. nat., Corse 83-92.

376. Raichon C., Verneuil B. DE, Mol,énat M., i977. L'élevage du porc en Castagniccia. Ethnozootechnie (I6), 68-74.

377. REgaudie R., Reveleau L., I977. Le mouton. J.B. Baillère, Paris, 282-283. 
378. SAlVAT H., I977. Les aides aux investissements de modernisation des exploitations agricoles. Plans de développement. S.O.M.I.V.A.C.-SETCO (83), 25-28, 33-35.

379. S.E.I., I977. Réflexions sur le développement des activités agricoles à l'occasion du contrat de pays de la Castagniccia. C.N.R.A. Versailles.

380. S.O.M.I.V.A.C., I977. L'élevage bovin en Corse. Ministère de l'Agriculture, Région Corse, ronéoté $59 \mathrm{p}$.

381. Sowa E., Cuenca J. C., 1977. L'utilisation du maquis dans les communautés rurales du Filosorma. Bull. Soc. Sci. hist. nat. Corse (625), 75-82.

382. S.U.A.D., I977. Proposition pour une politique de l'élevage. Chambre d'Agriculture d'Ajaccio, 20 p. ronéoté.

383. Viale Denise, 1977. Essai d'appréciation des potentialités alimentaires du maquis. Bull. Soc. Sci. hist. nat. Corse (625), 57-74.

384. Vissac B., I977. Conservation des races locales en liaison avec les potentialités du milieu naturel. Rapport du comité français au M.A.B., I 26-I 30.

385. VISSAC B., I977. Systèmes d'exploitation animale et valorisation des terres marginales boisées. Rapport du Comité français au M.A.B., 76-78.

\section{978}

386. Amandier L., Janin Edith, 1978. Améliorations pastorales et prévention des incendies. Parc naturel régional de la Corse, $32 \mathrm{p}$.

387. Assens Anne-Marie, 1978. Aspects de la vie d'une communauté rurale en Balagne de 1790 à 1920 : San Antonio. Mémoire de Maîtrise, Aix-en-Provence.

388. Astrier A., 1978. Le développement agricole et rural de l'intérieur de la Corse. Ministère de l'Agriculture, Région Corse, Ajaccio, $329 \mathrm{p}$.

389. BOREL-LFANDRI J. M., I978. Architecture et vie traditionnelle en Corse. S.E.R.G.

390. Bunch T. D., N'Guven T. C., Lauvergne J.J., 1978. Hemoglobins of the Corsico-Sardinian Mouflon (Ovis musimon) and their implications for the origin of $H b A$ in domestic sheep (Ovis avies). Ann. Génét. Sél. anim., 10, 503-506.

39r. CARDI Laurence, I 978 . Les transformations de l'élevage pastoral dans les vallées du Fango et du Marsolino. A.P.E.E.M., 20 p. ronéoté, Bastia.

392. Casanova A., I978. Techniques et sociétés. Forces productives et rapports de production dans les communautés rurales corses (fin XVIII - début XIXe siècle). in CAIsson et al. Pieve e Paesi, C.N.R.S. Paris, 33-69.

393. C.E.P.E., G.R.E.S.E., I978. Recherches sur les conditions agro-écologiques et sociales du développement du potentiel herbager et animal de la commune de Sollacaro et de l'ensemble de la vallée du Taravo, Corse du Sud (per. été r 977 aut. 1978). C.E.P.E., G.R.E.S.E. Montpellier.

394. CERutTi J., I978. La station d'élevage d'Altiani : an Quatre S.O.M.I.V.A.C.-SETCO, Avril, 33-42.

395. Colombani P., I978. Roquefort en Corse - Quatre laiteries et cinq caves maintenues en exploitation. Nice-Matin, 26-XI.

396. Cristofini B., Deffontaines J. P., Raichon C., Verneuil, B. DE, Joffrin J., LEvivieir M. C., Mercier O., I 978 . Pratiques d'élevage en Castagniccia. Exploration du milieu naturel et social en Corse. Étud. rurales (7 I-72), 89-I 09 .

397. D.D.A., 1978. Frissons de fièvre. Kyrn, (9I) 25-26. (D.A. pour Dominique ANTONI).

398. D.D.A. DE LA CORSE, 1978. Le développement agricole et rural de l'intérieur de la Corse. Ajaccio.

399. DEFRANCESCHI J., 1978. La communauté rurale corse à la fin de l'ancien régime : recherches sur l'occupation des sols. in Pieve e Paesi, C.N.R.S., Paris, 79-103.

400. Denis B., LAUVERGNe J. J., Théret M., 1978. Un variant clair du Mouflon Corsico-sarde (Ovis musimon) dû à un allèle au locus $B$ (Brun). Ann. Génét. Sél. anim., 10, 507-515.

40r. Franceschi P., I978. Étude cytogénétique du porc corse (Sus scrota domesticus L.) D.E.A. de Biologie évolutive des populations et des espèces animales. Univ. Paris VI, I 3 p., ronéoté.

402. GrUhIER F., 1978. Des calories à tort et à travers. Le Nouvel Observateur, 9/1/78, 52 .

403. KYRN, I978. 500 brebis et des problèmes. Kyrn (83), 40.

404. LaUvergne, J. J., HowelL W. E., I978. Un premier inventaire génétique de la chèvre Corse (gènes à effets visibles). Ethnozootechnie (22), 86-93.

405. LECA J. C., I 978 . Une communauté villageoise au XIXe siècle : Calenzana. In Pieve $\mathrm{e}$ Paesi, C.N.R.S., Paris, I05-133. 
406. Lenclud G., Pernet F., 1978. Ressources du milieu, gestion du troupeau et évolution sociale : le cas de la Corse. Étud. Rurales (71-72), 49-87.

407. NICE MATIN, 1978. Le M.O.D.E.F. et Roquefort. Ntce Matin, nov., Nice.

408. PERNET F., 1978. Quelques questions exemplaires à propos du pastoralisme Corse - Peuples méditerranéens, Paris (2) (janv-mars) 73-85.

409. POMPONi F., I978. Populations, structures agraires et production en Corse. (1770-1914) Contribution à un atlas historique de la Corse. In "Pieve e Paesi ", C.N.R.S., Paris 269-363.

4IO. Raulin H., Ravis-Giordani G., 1978. L'architecture rurale française : Corse. Berger Levrault, Nancy - 253 p.

4I I. Ravis-Giondani G., r978. Compte rendu de F. Pernet et G. Lenchud. Berger en Corse. Essai sur la question pastorale. Études Corses (9), I43-146.

4I 2. RAvis-GioRdani G., I978. Espaces et groupes sociaux : organisation objective et appréhension symbolique. In CaISson et al., Pieve et Paesi. C.N.R.S., Paris, I35-153.

4I3. S.E.T., I 978 . Utilisation des parcours par les bergers de la Castagniccia. Xemes Journées du Grenier de Theix. $18 \mathrm{p}$. ronéoté.

414. S.U.A.D., 1978. Programme pluriennal de développement agricole : Haute Corse 19781980. Chambre d'Agriculture de la Haute Corse, Bastia, ronéoté.

4I5. VALÉ́RY C., 1978. Élevage; la longue marche. Kyrn (87), 39-4I.

4I6. Verneuil, B. De, Raichon C., DefFontaines J. P., i978. L'amélioration de la production fourıagère en Corse intéresse-t-elle les éleveurs? Fourrage, (75) 3-28.

\section{I979}

4I7. Amandier L., I979. Bases phyto-écologiques pour un zonage agro-sylvo-pastoral de la Corse. Journées Scientifiques Ecologie et développement, C.N.R.S., I9-20 Sept. Paris, I 5 p. ronéoté.

4I8. ANTON D., I979. Des chercheurs de l'I.N.R.A. affirment la "cohérence" des méthodes traditionnelles d'élevage par rapport aux orientations modernistes. Le Provençal, Mars.

419. Cristofini B., Deffontaines J. P., Raichon C., Verneuil, B. DE, I979. Piatiques d'élevage et races en Castagniccia. Ethnozootechnie (24), $4 \mathrm{I}-52$.

420. Ertori F., Pomponi F., Ravis-Grordani G., Simi P., Pecoueux R., Babbont, Renucci Janine, I979. Corse : Écologie, Économie, Art, Littérature, Langue, Histoire, Traditions Populaires, Christine BONNETON, 87-126, 269-284.

42I. Grison P., i979. Compte-rendu général d'activité de l'unité de concertation sur l'écodéveloppement. I.N.R.A., La Minière, 55 p. rénéoté.

422. LaUvergne J. J., 1979. L'identité Corse se vérifie aussi chez les animaux. Kyrn (I), 24-26.

423. Molénat M., I979. Les zones marginales vont-elles infléchir la recherche porcine? Ethnozootechnie (24) 53-59.

424. Molénat M., CASAbianca F., 1979. Contribution à la maîtrise de l'élevage porcin extensif en Corse. Bull. techn. Dept. Génét. anim. I.N.R.A. France $\mathbf{n}^{\circ} 32,72$ p.

425. PÂTrE, I979. Ressources alimentaires du maquis Corse. Pâtre (262), 55-56.

426. Pezron (Monique LE), I979. Sollacaro I 788-I979, préalables à une approche socio-écologique du développement. Montpellier, i 8 p.

427. Poplin F., i979. Paléontologie du Mouflon corse dans la perspective nouvelle du marronage. Ann. Génét. Sél. anim., II, I33-143.

428. Salvat H., 1970. L'élevage bovin en Corse. S.O.M.I.V.A.C.-SETCO, (Janv.) 27-35.

429. TORRE Evelyne, Grisoni Marie-Josèphe, FlORI J., I 979 . Quelques aspects des communautés rurales corses au $\mathrm{XVI}^{\mathrm{e}}$ au XIX ${ }^{\mathrm{e}}$ siècle. C.R.D.P., Ajaccio. $57 \mathrm{p}$.

\section{NON DATÉS}

430. Branchemain A., La production caprine dans le Sud-Est. Cerater, 33 p.

43I. CANCELlifiri Madeleine. La vallée du Cruzzini.

432. Courtant M. L'évolution de la vie pastorale en Corse. S.D.A. Corse (dactyl.).

433. D.D.A. DE LA CORSE. La mise en valeur pastorale dans les régions d'économie montagnarde. 12 p. dact.

434. G.R. DE LA CORSE. Le programme d'abattoirs publics pour la Corse. Ajaccio. 


\section{Index des auteurs (I)}

Abbatucci S., 52.

ACQUAVIVA M., 260.

AdALsteinsson S., 334.

ALBERTINI J.-P., 236.

Albitraccia A., 96, 105.

ALIVI (L'), $280,348,349$.

ALSMARK G., 281 .

Amandier L., 386, 4 I 7 .

ANFossI G., 74 .

ANONYME, $25 \mathrm{I}, 282$.

Anton D., 418.

ANTONI D., 350 .

A.P.E.E.M., 26I

Archives départementales De r,a CoRse DU SUD, I, 4, 5, 7, 14, I6, I7, 18, 19, 21, $22,23,48,62$.

Archives DU Senat DE Gènes, 2.

ARChIVES NationaL,ES, 6, 9.

Ardouin-Dumazer, 65 .

A.R.E.E.A.R., 35 I

ARMANI V., I40.

ARrighi P., 215.

Assens Anne-Marie, 387 .

ASSOCIATION POUR LA GESTION DE IA Station D'Elevage D'Altiani, $283,3 \mathrm{I} 6$. AstiER A., 388.

AUJOURD'HUI LA CORSE, 237.

Aumaître A., 21 I.

BARROLI A., I 87 .

BATICLE Y., I 56, 262.

BARRÉ G., 195 .

BARRY J.-P., 284, 317.

B.D.P.A., 285,318 .

BELLON, 59.

BERENNI P., 263, 288.

BERGERAT E., 63.

BEVILACQUA M. V., 168.

BIgot M., 6 I

BLANCHARD R, 72.

BLANCHEMAIN A., 430.

BLANQUI F.A.U., 28 .

BIAASINI P., 238, 286.

BONNE A., 37.

BOREL-LEANDRI J.-M., 389 .

BORTOLAN M., IO4.

BOTTIGLIONI.

BOURGEAT F., 287.

BOYER L., $78,79,81,87$.

BRUGÈRE D., 319, 352 .

BUNCH T. D., 390.

BURNOUF, 43 .

Cabrot. I., 353.

CADORET H., 82.

Cancelrlieri Madeleine, $43 \mathrm{r}$.
Cantal (R. du), 55 .

Cardi Laurence, 391.

CARIOTTI J., IOO, IOI, I03, I57, I69.

CarlotTi R., 25, 33, 38, 39, 4I, 58, 60.

CARRIER J., 67.

Casabianca F., 354, 355, 424.

Casabjanca (F. de), 252, 356 .

Casanova A., 392.

CASTA T., 362 .

C.D.J.A. 264.

C.E.P.E., 349, 363, 393.

Cerutti J., I 88, 196, 225, 239, 394.

C.E.T.A., 264.

Chabaud A., 174 .

CHAMBRe DF́PARTEMENTALE D'AGRICUI,TURE, 264.

Charpentín B., 288.

Chiva I., I 33, 163.

Chorn (P. de), 73.

Claret R., I 89.

CODE CORSE, 8.

Colombani F., 226.

Colombani P. 395.

Colonna J. N., 289.

Comitr S., 89 .

Conrad Marcelle, 253, 254, 290, 320.

CoTTE M. A., 216.

Courtant M., 432.

C.P.S., 224, 265.

CRESPO D. G., 357

Cristorini B., 396, 4 I 9.

C.T.C.E.P., III.

Cunnca J. C., $3^{81}$.

D.A., 397 .

DAJOZ R., 205.

D.A.T.A.R., $24^{\circ}$

D.D.A. DE LA CORSE, I 59, I 70, I75, I 76 , I77, 197, 206, 207, $212,217,227,229$, 24I, 249, 29I, 3II, 398, 433.

DEFFONTAINES J.-P., 352, 396, 4I 6, 419.

DEFRANCESCHI J., 266, 399.

Degos G., I I 8, I 23, I 24, I42, 160.

DEL,AHAYE J., 358 .

DELOGU M. R., I 78 .

DELVARRE G., 263.

DENIS B., 364,400 .

DOCUMENTATION FRANÇAISE, I $4 \mathrm{I}$.

D.S.V. DE I,A CORSE, 229.

DUMONT R., I I 3, I I 4 , I I 5 .

Dupias G., I6I, 179 .

ÉCOCHARD M., 2 I 4.

E.D.E. CORSE, 267

ENDERLIN J.-P., 359.

ENQUÊTE AGRICOLF, 53.

(I) Les cotes sont celles de la liste chronologique. 
ESCARD F., 6I.

ÉTIENNE M., 292, 293, 294, 295, 322, 323, $324,325,326,327,360$.

E'TTORI F., II9, I 28, 420.

EXPOSITION A.T.P., CORSE, 268.

FALCUCCI C., 230.

FaUCher D., 83.

FÉdÉRATION OVINE DE CORSE, 296.

FERTON C., 70.

FEXDEL, 12 .

Figaro agricole (le), i 98.

FIZELIERE (de la), ro.

Framant J.-C., 213, 252, 297, 298, 299, 371 .

FLORI F., 361 .

FLORI J., 362, 429.

F.N.S.E.A., I99, 264.

Foata (Abbé de la), 45.

FRANCESCHI P., $40 \mathrm{I}$.

Franceschini J., 109.

GaLIANI Jeanne, 2 I 8.

Gamisans J., 328.

GARDELLE C., 2 I 9

GaUdo'T G., 92 .

Gaussen H., I 79.

GAVINI A. J., 29.

GENSOLLEN J.-P., I 44 .

GERI C., 300, 329.

Grese (W. von), 9I

GINDRO J.-C., 33 o.

GIRAUD-GENET L., 7I, 8o.

GoTO N., 2 I 4.

G.R. DE LA CORSE, 434.

GrFE J.-P., 353.

G.R.E.S.E., 349, 363, 393.

GRISCEL,LI DE VEZZANI J.-F., 46.

GRISON P., 424, 421.

GrIsonI Marie-Josèphe, 429.

GRUHIER F., 402.

GUF́RIN G., 269.

Guiard-Marigny Hélène, 220.

Guilaine J., 33 I.

G.V.A., 264 .

HERCHER P., I 34, 200.

HOWELL W. E., 404.

Hudault C., I 20.

IZARD M., I 79.

JANIN Édith, 386 .

JEAN BRUHNES-DE LA MARRE Mariel, I 49.

JEANNIN A., I 6 .

JEANNIN B., I I 7 .

JOFFRIN J., 396.

JOLEAUD L., 86.

KELLER M., I 35, I 36.

KOLODNY Y., I5O.

KYRN, 403 .
L.ACOSTE A., 332 .

LAIEURE J.-P., 242.

I.AMOTTE P., I 25, I 26, I 29, I62.

LANFRANCHI (F. de), 255, 30I.

LANGLET B., 263.

LARUE P., 66.

LAURANS R., 302, 303.

LAURENT, 84 .

I.AUVERGNE J. J., 304, 333, 334, 364, 390, $400,404,422$.

LECA J.-C., $270,405$.

LEENHARDT M., $23 \mathrm{I}$.

LEFEBVRE P., I 21 .

LEJEAI, J.-P., 243.

LENCLUD G., 256, 300, 335, 336, 374, 406.

LEROY A. M., IIO.

LETELLIER, 2 I 6.

LEVIVIER M. C., 396.

Libro Delie PiANTE, 3.

LIMPERANI (J. A. de), 50, 54.

LONG G., 365,366 .

LuCraNi C., I 5I, 367 .

LUCIANI F., 368.

LUCIEN C., 233.

LUCKE H., 337.

M.A.B., 369.

MAESTRONI-MEGLIA, 47.

MAGDENEL Évelyne, $27 \mathrm{I}$.

MaIsani M., I 80 .

Maniccia P., 244.

MANIÈRE R., 284, 317.

Marchi G., 40.

Martini M., I63.

MASON I. L., 201 .

MASSIGNon Geneviève, I64, I $8 \mathrm{r}$.

MAUGini A., 88.

MÉNASSEYRE, I 30 .

Mercier O., 396.

MERIA G., 22 I.

MERIMÉE P., 30 .

MEYER-HEINE G., I 3 I .

MICALEFF P., 2 I 6, 232.

Michel Chantal,

M.I.P.A.E.N.M., 338, 339, 370.

MIQUET J., 208.

M.L./M.B.T., 305 .

MOLENAT G., 252, $37 \mathrm{I}$.

MolénAT M., $340,355,372,376,423,424$.

MOLINIER R., I 37 .

MOLL L., 27.

MONTARD F. de, 257

MORANDIÈrE (C. de la), 97

Mouton (Le), I 86.

MusÉE National A.T.P., I02.

Natali J.-B., 76, 90, 93, 98.

Navarko J., 362 .

NEUWIRTH M., I 45 .

N'GUYENG T. C., 390.

Nice Matin, 407.

NivaggionI T', $25^{8}$.

Nizza P. F., 190. 
OGIER J., 34I.

O.N.F., 259

OTtavi G.A., 56 .

OTtaVi P., 146.

PALÉl,ogue G., 24 .

PALMIERI M., 203.

PANIS .M., I 38 .

PAOLETTI M., I 82, 244.

PÂTRE, 373, 425 .

PeCQUEUX-BARBoni R., 420.

PeRFETtini M., I9I.

Pernet F., 272, 336, 374, 406, 408.

PERRET G., 233.

PERRIER E., 273

Petit Bastiais (le), 94.

Peyraúd D., 233.

Pezron (Monique le ), 426.

PFEFFER P., I 65, 202.

PieretTi A., I07, I 12.

PiETRI J., 362.

Pietry A., 13.

Pichód-Viale Denise, 222.

PIOBB P. V., 75.

PITTI FERRANDI A., 306.

Plan TERrier de la Corse, i I.

POLI, 4.4.

PorI Gisèle, 307.

POMPET P., 77.

Pomponi Janine, I 52.

Pomponi F., 274, 275, 308, 342, 409, 420.

POPLIN F., 430, 427 .

POZzo DI BORGO C. A., 34, 57.

PRALORAN J.-C., 2 I 4, 245.

PrasseTO, $5 \mathrm{I}$.

PrÉfecture DE CoRse, 309.

Programme D'Action RÉGIONAle CORSE, I 22.

PRUd'HON M., 37 I.

QUiTTET E., I66, I83, 234.

RACON S., 276, 343, 375 .

RAICHON S., $35^{2}, 376,396,416,419$.

RAULIN H., 4IO.

RAVEL, L., 68.

Ravis-GIORdANI G., I $84,246,256,310$, 4IO, 4II, 4I2, 420.

REGAUDIE R., 377.

RENUCCI Janine, 223, 235, 277, 420.

RENUCCI T.,

REPARAZ (G.A. de), I47, I 53.
REVELEAU L., 377.

REY P., I 79.

ROBIQUET F., 26.

ROMANI, I 71 .

RONDEAU A., 172.

ROSARI J., 99.

Sajous P., 79, 81, 87 .

Salvat M., 378,428 .

SANSON A., 69,

SARRE M., 203.

S.C.E.E.S., 247, 248, 249, 3 II.

S.C.E.T., 259.

S.E.I., 3I 2, 344, 345, 379, 4I3.

Silvagnot,A A., 192.

Simi P., I 32, I 54, I93, 278, 420.

S.O.D.E.T.E.G., 3 I 8.

S.O.M.I.V.A.C., $148, \quad 155,173, \quad 185,346$, 380 .

SowA E., $38 \mathrm{I}$.

SPINOSI F., I 43

S.R.S.A., 279.

Statuti Civile e Criminali di Corsica, 32.

S.U.A.D., 3I3, 382, 4 I 4 .

TERIGI P., 49.

TESSIER C., I 5 .

TESTANIER J., I 39.

THERET M., 364,400 .

Thiaulit M., $37 \mathrm{I}$.

TORRE Évelyne, 429.

TOUSSAINT G., 209.

Tranti N., 108.

VALÉRY C., 415.

VATTI G., 95.

VERNEUII (B. de), 314, 340, 352, 372, 376, $396,416,419$.

Viale Denise, 315, 347, 383 .

VIANGALLI Jeanine, I 27.

VIGAROUS, 20.

VILIAAT L., 85

VINCIGUERRA S., 167 .

VISSAC B., 384,385 .

VIVAS J.-P., 250.

VUILLIER G., 64 .

WEIPERT A., 35, 36 .

WEISS M. C., I94, 255, 301 .

WIID M., 204.

ZERT P., 234.

\section{Index des matières}

Abattoirs, Viande, I 7I, 434 (I).

ANE.

ARCHÉoLOGIE, 255, 301, 331.
ARCHITECTURE, TRADITIONNELLE, 389, 4 IO. Aviculture, 203.

Bovins (Corses), 17, 43, 69, 166, I73, 380 .

(I) Les cotes renvoient à la liste chronologique. 
Bovins (Tarentais, 82, 428 .

CAPRINS, 19, 38, I 56, 182, 208, 209, 2 1 0, 237, 276, 307, 346, 368, 404, 430.

Chevaux, $16,43,55,73$.

CHIEN, 302.

Charcuterie, 372 .

Comparaison avec I A Sardaigne, 95, II4, 304.

CoNCOURS, 50.

CONSERVATION DES STOCKS GF́nÉtiques, $304,333,384,422$.

CONSOMMATION, 48,62 .

DÉMOGRAPHIE DES EXPLOITANTS, 74, 121, I 76, I 77, 229, 244 .

DoLÉANCES, 46, 273, 275, 397, 407, 415 .

ÉTUDES LOCALES ET RÉGIONALES :

Agriates, I 12, 163 .

Antisanti, 317 .

Asco, 192 .

Balagne, 185,188 .

Bastelica, 6I, 258.

Cap Corse, 286.

Casabianda, 136.

Calenzana, 405 .

Casinca, $23^{\circ}$.

Castagniccia, 221, 269, 312, 319, 344, $345,35^{2}, 35^{6}, 37^{6}, 379,396,4^{1} 3$, 4 I 9.

Castifao, 5, 250 .

Corse, I 5, 20, 24, 26, 27, 28, 30, 31, 33, $40,5 \mathrm{I}, 54,55,56,57,59,64,65,66,67$, $68,75,77,84,85,88,94,96,99$, 100, 101, 102, 103, 104, 106, I13, 128, I41, I 43, I 72, 199, 277, 309, 420 .

Corse (Haute), 4 I 4.

Corse orientale, 9, 29.

Corse du Sud, 89, 35 I.

Coscione, 90, 98, $13^{8}$.

Coti Chiavari, 218 .

Cruzini, 43I.

Dépression centrale, I93.

Filosorma, 320, 332, 343, 347, 375, 38 r, $39 \mathrm{I}$.

Fozzano, 125.

Golo (Departement), I3, I4.

Golo (Vallée), 263, 287, 292, 293, 294, 295, $322,323,324,325,326,338,339,34 \mathrm{I}$.

Gradule, I94, 242.

Gravone, 242 .

Luri, 236.

Nabbio, 132.

Niolo, 9I, 93, 97, I 35, I 39, I40, I42, 238, 310.

Plaine orientale, 107, I 19.

Porto, I 68.

Prunelli, 242.

San Antonio, 387 .

Sartenais, I0, 265 .

Serra di Scopamene, 152.

Sollacaro, $280,349,363,426$.
Sotta, I 53

Taravo, I09, 348, 393 .

Tavignano, 284 .

Ucciani, 330.

Vescovato, 337 .

Vico, $27 \mathrm{r}$.

FaUne SAUVAGE, 86, 222.

FLORE, ETHNOBOTANIQUE, I 37, I 61, I 79, $254,290,317,3$ I $8,320,32$ I, 4 I 7.

Fromages, $37,49,70,78,186,189,289$, $305,373,395$.

INCENDIES, 253, 325, 338.

LÉGISLATION, 2, 3, 4, 7, 8, 3I, 47.

MAQUIS, I 24, 26I, 278, 282, 31 $5,320,32 \mathrm{I}$, $332,369,381,383,425$.

MCEURS DES BERGERS, A.T.P., 1 $2,72,76$, 108, I49, I62, I64, I67, I $81,184,215$, $266,268,353,359,429,432$.

MOUFI.ONS, 63, 86, I65, I 74, 202, 205, $364,390,400,427$.

Ovins, 22, 44, 79, 81, 83, 87, 92, I10, I 23, I 36,146, I $56,169,178,183,188,189$, $195,201,213,220,226,231,233,243$, $262,279,283,306,311,316,334,367$, $377,394,403$.

PlaNS DE RECHERCHE ET DE DEVELOPPEMENT, 25, 34, I I I, I I 5, I I6, I I 7, I 1 8, I 20, I 22, I 30, I 3 I , I 33, I 34, I 39, $145, I_{4} 8, I_{5}, I_{54}, I_{55}, I_{75}, I_{85}, I_{87}$, $214,238,239,240,252,256,259,260$, $264,267,286,287,288,291,292,293$, 294, 295, 296, 297, 298, 299, 300, 306, $313,324,327,339,341,349,351,352$, $365,366,370,371,378,379,382,388$, $398,414,421,427,433$.

Plan TERRIER, II, 105.

Porcins, I90, 197, 207, 21 I, 212, 217 , $224,228,234,24$ I, 245, 340, 354, 355, $376,401,423,424$.

Prairies CUltivées, AMÉlioration DES PARCOURS, 6, I 70, I96, 200, 204, 216, $225,232,257,314,325,326,327,338$, $357,360,36 \mathrm{r}, 386,4 \mathrm{I} 3,4 \mathrm{I} 6$.

PROPHYLAXIE, 358 .

SF́RICICULTURE, $35,36,60$.

SONNAILLES, 303.

STATISTIQUES, 23, 52, 53, I75, 247, 248, 249. SYSTEMES D'AGRICULTURE ET D'ÉLEVAGE, I4, 7 I, I I 3, I 26, I 27, I 44, I 47, I 53, I 50, $157,158,160,180,198,206,219,220$, $223,227,235,246,251,272,281,285$, $310,329,335,336,337,350,362,374$, $385,392,396,399,402,406,408,409$, $4 \mathrm{II}, 4 \mathrm{I} 2,4 \mathrm{I} 8,4 \mathrm{Ig}$

Traités D'Ágriculture ET D'ÉLEVAGE, 42.

VaINe pature, Bifins Communaux, CloTURES, I, 2, 4, 7,8, I8, 20, 39, 4I, 45, $46,58,80,129,274,308,342$. 


\section{Discussion}

\section{Insuffisances et lacunes}

On note tout d'abord qu'une grande partie des références concernant l'année I979 manquent. On en dénombre en effet seulement I3 alors qu'en I977 et I978, 37 et $3 I$ cotes respectivement ont été recensées. Évidemment début I 980 il était impossible de réunir toutes les références de l'année précédente.

On pense également que par le passé, un certain nombre de références nous ont échappé car nous n'avons pas pu passer en revue avec assez d'attention les collections des journaux locaux ce qui exige évidemment un gros travail, ni les archives des D.D.A., des D.S.V., pas plus que les Archives Départementales (I) où Évelyne TORRE et al. (r979) signalent que la quête pourrait encore être fructueuse.

A noter ensuite qu'un certain nombre de références sont incomplètes : sans date (6), sans pagination, sans nom d'éditeur, sans adresse d'éditeur etc... ou erronées. Certaines de ces lacunes pourront être complétées grâce à des recherches plus approfondies et comme pour les références manquantes, on prie instamment tous les lecteurs de nous excuser et de nous aider à rétablir la vérité.

\section{Bilan global}

On a recensé 434 cotes dont les trois quarts datent des 30 dernières années (entre I948 et I979) et la moitié remonte seulement au plus à I970. Au total 3I2 différents auteurs ont contribué à ces travaux.

L'histogramme de la figure I qui porte sur le $\mathrm{xx}^{\mathrm{e}}$ siècle montre une progression à allure exponentielle (à part l'accroc des années quarante dû au

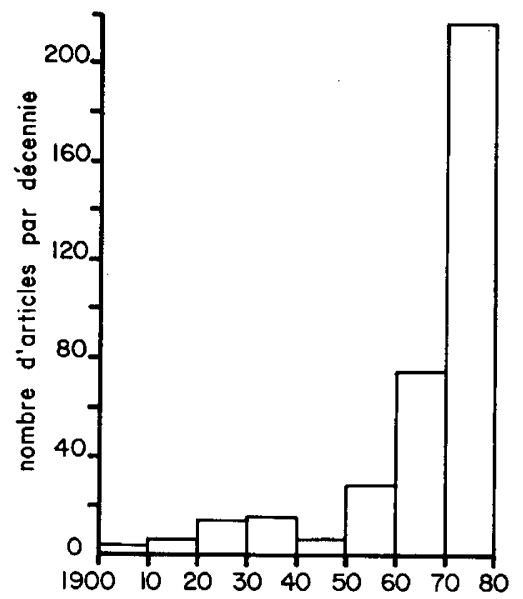

FIG. I. - La progression du nombre des articles consacrés à l'élevage en Corse de décennie en décennie depuis rgoo.

The progress of the number of articles derated

to annual husbandry in Corsica by decade since Igoo.

(I) Anciennement pour toute l'île, à Ajaccio, cet urganisme est maitenant devenu Archives Départementales de la Corse du Sud. 
conflit mondial) avec un éclatement qui s'amorce dans les années cinquante pour se réaliser dans les années soixante et surtout soixante-dix.

I1 est probable que l'adjonction des articles manquants ne modifiera que faiblement ce tableau évolutif que nous avons également observé récemment dans le domaine de la conservation des stocks génétiques en France (I A AUVERGNE et LAURANS, I979).

\section{Évolution qualitative}

Ce n'est qu'à la fin du xvmI $^{\mathbf{e}}$ siècle que nous trouvons les premières références consacrées à l'élevage dans le but de l'améliorer et de le connaître (Réf. no 6 et 9). Auparavant, les documents que l'on trouve sont en quelque sorte involontairement consacrés à l'élevage; il s'agit en premier lieu de régler des problèmes de vaine pâture et de clôture qui étaient une source de conflits parfois graves dans l'ancienne société corse.

Au courant $\mathrm{du} \mathrm{xIX}^{\mathrm{e}}$ siècle, les rapports généraux vont alterner avec des statistiques, cependant que la question du droit de pacage est encore souvent évoquée.

Au début du $\mathrm{xx}^{\mathrm{e}}$ siècle la situation est peu changée mais les études spécifiques et les monographies locales font leur apparition.

Dans les années cinquante, où se situe le début de l'éclatement quantitatif, on assiste à la multiplication des monographies et des études d'aménagement et cette situation s'accentue au cours des années soixante où les études deviennent plus spécialisées et plus documentées préludant aux séries d'études des années soixante-dix : espèces, parcours naturels, maquis, prairies artificielles, prévention des incendies, études historiques de petites régions, études scientifiques débouchant sur le développement de certaines autres avec en particulier les monographies sur la Castagniccia, le Filosorma, le Taravo, le Niolo qui sont des modèles du genre.

\section{Les causes de l'évolution}

Après la guerre en France de plus en plus l'aide gouvernementale s'étend sur tous les secteurs, en particulier l'agriculture. Pour la Corse, cela se traduit par l'aménagement de la plaine orientale, coordonné par la S.O.M.I.V.A.C. (créée en 1957$)$.

Tout d'abord purement agricole (vigne, agrumes) cette action s'oriente vers l'élevage jusqu'alors parent pauvre d'où l'activité qui démarre dans les années soixante et s'accélère dans les années soixante-dix. Il appert en outre assez vite que le développement de l'élevage doit être considéré d'un point de vue écologique ne serait-ce que pour essayer de réduire les incendies par un équilibre sylvopastoral bien compris.

Ainsi, compte-t-on dans les années soixante-dix des programmes de recherches et de développement qui sont lancés par une quarantaine d'organismes locaux et en général publics ou para publics ou syndicaux $\left({ }^{1}\right)$ : D.D.A., S.O.M.I.V.A.C., C.P.S., G.V.A., ... mais aussi de statut privé comme 1'A.P.E.E.M., ou également continentaux et à but purement scientifique comme les Universités qui envoient leurs thésards, le C.N.R.S. avec le C.E.P.E. ou 1'I.N.R.A. etc...

(I) Cf p. 9i pour la liste de ces organismes. 


\section{Problèmes de documentation posé par la présente bibliographie}

L'appel des références en mode conversationnel sur les fichiers internationaux déjà existant a été peu fructueux : I 7 références depuis 1972 alors que nous en citons I94. La cause en est 1'abondance des entrepreneurs de recherche et de développement que nous venons d'évoquer car très souvent, ces activités se soldent par une publication interne que ces centres se soucient peu de déposer dans les bibliothèques intéressées.

Avec les moyens modernes, il existe cependant un remède qui consisterait à microficher systématiquement les références non périodiques et à mentionner à la suite de la référence elle-même l'indicatif de la bibliothèque où elle se trouve.

A noter que ce procédé pourrait être utilisé avec profit pour toute la série des ouvrages anciens non périodiques pour la plupart eux aussi introuvables.

\section{Conclusion}

Le nombre des travaux écrits consacrés à l'élevage en Corse s'est beaucoup accru ces deux dernières années. Cet accroissement quantitatif s'accompagne d'un éclatement disciplinaire remarquable.

Un renforcement de l'efficacité de certaines bibliothèques semble nécessaire afin de rendre ces documents facilement accessibles. Des revues par disciplines semblent également nécessaires.

\section{Remerciements}

Cette compilation n'aurait pas été possible sans l'aide des personnes dont les noms suivent :

- Mme Maryse Gautier et M.M. Long et Thiaulir du C.E.P.E. Montpellier.

- M. A. Salasca, Chambre d'Agriculture, Ajaccio.

- Mme Denise VIaLE, A.P.E.E.M., Bastia.

- M. HÉlion, Centre de Documentation de la M.N.E. Paris.

- M. François FLoRI, Bastia.

- M. D. BRUGËE, S.E.I.-C.N.R.A., Versailles (maintenant S.A.D.E.).

- M. R. Laurans, Société d'Ethnozootechnie, Paris.

- M. J.N. MatTeI, Bibliothèque du Ministère de l'Agriculture, Paris.

- M. E. Grison, Bibliothèque de l'E.N.V. Alfort.

- M. M. MOLÉnat, Département de Génétique animale, C.N.R.Z., Jouy-en-Josas.

- Mme Catherine IBorRA, Documentation centrale, C.N.R.Z., Jouy-en-Josas.

- M. P. Lamotre, Direction des Services d'Archives de la Corse du Sud, Ajaccio.

- M. H. RouveYrol, C.R.D.P., Ajaccio.

\section{Summary}

\section{Animal Husbandry in Corsica : a descriptive Bibliography}

Between 1562 and 1979,434 references devoted to animal husbandry in Corsica have been recorded. They have been produced by 3 I 2 different authors. Three quarters of the quoted papers trace back to thirty years and half of them have been written these last ten years. This recent and pronounced acceleration is resulting from a governmental pressure in order to improve agriculture, economy and more recently ecologic situation of the island. The more recent works whose quality is in constant progress are originating from about forty different organisms. 


\section{Références bibliographiques}

BrUGÈrE D., I974. Bibliographie générale de la Corse. Éléments sélectionnés. I.N.R.A., S.E.I., Versailles, ronéoté, $19 \mathrm{p}$.

ETrENNE M., 1977. Bases phytoécologiques du développement des ressources pastorales en Corse. Thèse, Université de Montpellier, 2 ro p.

FAURE R., 1978. Le Dictionnaire de l'Agriculture. Centre de documentation et d'information rurale, Paris, so éd., 431 p.

Flori F., r974. Bibliographie générale de la Corse, année 1972. Centre d'Études Corses d'Aixen-Provence, $30 \mathrm{p}$.

FıORI F., I975. Bibliographie générale de la Corse, années I973, I974. Centre d'Études Corses. Aix-en-Provence, $47 \mathrm{p}$.

FlORI F., I976. Bibliographie générale de la Corse, année i 975 . Operata culturale Corsa, Corti, $29 \mathrm{p}$.

LAUVERGNe J. J., LAURANS R., I979. Inventaire et conservation du matériel génétique animal de ferme en France et écodéveloppement : une bibliographie signalétique I96I-I969. Ann. Génét. Sél. anim., 11, 165-185.

Simi P., r966. L'adaptation humaine dans la dépression centrale de la Corse. Thèse de géographie humaine, Aix-en-Provence, Orphys, Gap, 260 p.

Strarace C., r943. Bibliographia della Corsica. Istituto per gli studi di politica internazionale, Centro di Studi per la Corsica.

TORrE Evelyne, Grisoni Marie-Josèphe, FLORI J., I979. Quelques aspects des communautés rurales corses du $16^{\mathrm{e}}$ au $19^{\mathrm{e}}$ siècle. C.R.D.P. Ajaccio, $57 \mathrm{p}$. 